\title{
Numerical Methods and Software Tools for Simulation, Design, and Resonant Mode Analysis of Radio Frequency Birdcage Coils Used in MRI
}

\author{
NECIP GURLER, YUSUF ZIYA IDER \\ Department of Electrical and Electronics Engineering, Bilkent University, Çankaya, Ankara 06800, Turkey
}

\begin{abstract}
Design of magnetic resonance imaging (MRI) radiofrequency (RF) coils using lumped circuit modeling based techniques begins to fail at high frequencies, and therefore more accurate models based on the electromagnetic field calculations must be used. Field calculations are also necessary to understand the interactions between the RF field and the subject inside the coil. Furthermore, observing the resonance behavior of the coil and the fields at the resonance frequencies have importance for design and analysis. In this study, finite element method (FEM) based methods have been proposed for accurate time-harmonic electromagnetic simulations, estimation of the tuning capacitors on the rungs or end rings, and the resonant mode analysis of the birdcage coils. Capacitance estimation was achieved by maximizing the magnitude of the port impedance at the desired frequency while simultaneously minimizing the variance of RF magnetic field in the region of interest. In order for the proposed methods to be conveniently applicable, two software tools, resonant mode and frequency domain analyzer (RM-FDA) and Optimum Capacitance Finder (OptiCF), were developed. Simulation results for the validation and verification of the software tools are provided for different cases including human head simulations. Additionally, two handmade birdcage coils (low-pass and high-pass) were built and resonance mode measurements were made. Results of the software tools are compared with the measurement results as well as with the results of the lumped circuit modeling based method. It has been shown that the proposed software tools can be used for accurate simulation and design of birdcage coils. (c) 2015 Wiley Periodicals, Inc. Concepts Magn Reson Part B (Magn Reson Engineering) 45B: 13-32, 2015
\end{abstract}

KEY WORDS: RF birdcage coil; coil design; electromagnetic simulation; resonant mode analysis; software tool; finite element method; high-field MRI

\section{INTRODUCTION}

Magnetic resonance imaging (MRI) systems with higher static magnetic field $\left(B_{0}\right)$ have higher signal

Received 18 July 2014; revised 15 April 2015; accepted 20 April 2015

Correspondence to: Yusuf Ziya Ider; E-mail: ider@ee.bilkent. edu.tr

Concepts in Magnetic Resonance Part B, Vol. 45B(1) 13-32 (2015)

Published online in Wiley Online Library (wileyonlinelibrary. com). DOI: 10.1002/cmr.b.21279

(c) 2015 Wiley Periodicals, Inc. to noise ratio (SNR) among other advantages. However, the use of high-field strengths $(\geq 3 \mathrm{~T})$, and hence high radiofrequencies (RFs), brings challenges to the area of RF coil design. For example the traditional lumped circuit element model based techniques (1-6) used in RF coil design become unreliable since the quasi-static approximations made in such models begin to fail at high frequencies. Therefore more accurate methods, which are based on the numerical calculation of the electromagnetic fields, are required in designing RF coils. In the literature, many applications based on the numerical electromagnetic field calculations have 
been proposed. These applications use finite difference time domain method (FDTD) (7-13), finite element method (FEM) $(14,15)$, method of moments (MoMs) (16-18), or hybrid numerical techniques (19-22).

In addition to being used for more accurate coil designs, electromagnetic field calculations are also used to understand and interpret the interactions between the RF field and the subject inside the coil, which are more complex at high frequencies and affect image quality (23-25). For instance electromagnetic field calculations are very important for " $\mathrm{B}_{1}$ shimming" techniques which aim at obtaining a relatively homogenous RF field distribution in the region of interest (ROI) (26-29). Furthermore, electromagnetic field calculations can also be used for safety analysis, such as obtaining local specific absorption rate (SAR) distributions in order to predict the presence of hot spots $(10,11,15,19,30,31)$.

In this study, FEM based methods have been proposed for accurate simulation, design, and resonant mode analysis of low-pass and high-pass RF birdcage coils using COMSOL Multiphysics (COMSOL AB, Stockholm, Sweden), a FEM based software package. For design purposes, an optimization based new method was developed to calculate the capacitance value, which makes the coil resonate at the desired frequency. In addition to capacitance calculation, this study presents direct resonant modes analysis based on the time-harmonic electromagnetic formulation of the problem. This analysis is important to make sure that the homogenous mode is far away from the other modes so that tuning of the coil can be done without interfering with the other modes (5). Furthermore, resonant mode analysis can also be used to discriminate the homogenous mode of the double-tuned birdcage coils, which may overlap with the higher modes of the inner birdcage coil (32). Another example of the use of resonant mode analysis is the determination of the end-ring resonant mode (or Helmholtz mode) of a high-pass birdcage coil used in a vertical bore MRI system (5).

\section{Abbreviations}

$\begin{array}{ll}\text { CSF } & \text { cerebrospinal fluid } \\ \text { FDTD } & \text { finite difference time domain method } \\ \text { FEM } & \text { finite element method } \\ \text { GM } & \text { grey matter } \\ \text { MoMs } & \text { method of moments } \\ \text { MREPT } & \text { magnetic resonance electrical properties } \\ & \text { tomography } \\ \text { MRI } & \text { magnetic resonance imaging } \\ \text { RF } & \text { radiofrequency } \\ \text { RM-FDA } & \text { resonant mode and frequency domain analyzer } \\ \text { ROI } & \text { region of interest } \\ \text { SAR } & \text { specific absorption rate }\end{array}$

In order to provide convenience to coil designers and researchers in the field of MRI in applying the methods proposed in this study, two software tools with graphical user interfaces (GUIs), resonant mode and frequency domain analyzer (RM-FDA) and Optimum Capacitance Finder (OptiCF), have been developed using MATLAB (The Mathworks, Natick). RM-FDA is used for two purposes. One of them is the calculation of the resonant mode frequencies and their associated fields. The other one is the computation of the time-harmonic electromagnetic field solutions for a specified range of frequencies. OptiCF, on the other hand, is used to determine the optimum capacitance value of a birdcage coil by using two objective functions, magnitude of the port impedance and the variance of the $B_{1}^{+}$magnitude in the ROI $\left(B_{1}^{+}\right.$is the MR-wise excitatory rotating component of the RF magnetic field). Both of these software tools are applicable to lowpass and high-pass birdcage coils, and the geometric information for all parts of the coils can be specified through their GUIs.

Simulation results for different scenarios obtained using the developed software tools are provided. To verify the results of the proposed software tools, OptiCF and RM-FDA, regarding capacitance values and resonant mode frequencies, two handmade birdcage coils (low-pass and high-pass) were constructed and experimental measurements were undertaken. The capacitance values obtained by OptiCF were also compared with the capacitance values found by BirdcageBuilder, which is a lumped circuit element model based design tool developed previously by Chin et al (6).

\section{MATERIALS AND METHODS}

\section{FEM Models of Low-Pass and High-Pass Birdcage Coils}

First, geometry of a birdcage coil was built in the simulation environment [Figs. 1(a,b)] (33). In this geometric model, rungs and end rings were modeled as $2 \mathrm{D}$ rectangular strips whereas lumped capacitors were modeled as 3D rectangular domains which have zero conductivity, free space permeability, and different permittivity [Fig. 1(c)]. The permittivity value of these rectangular domains was calculated for the given capacitance value using the parallel plate approximation.

After geometrically modeling the coil, boundary conditions were applied. Rungs, end-rings, capacitor plates and RF shields were modeled as $2 \mathrm{D}$ perfect electric conductors (PECs). For computational 

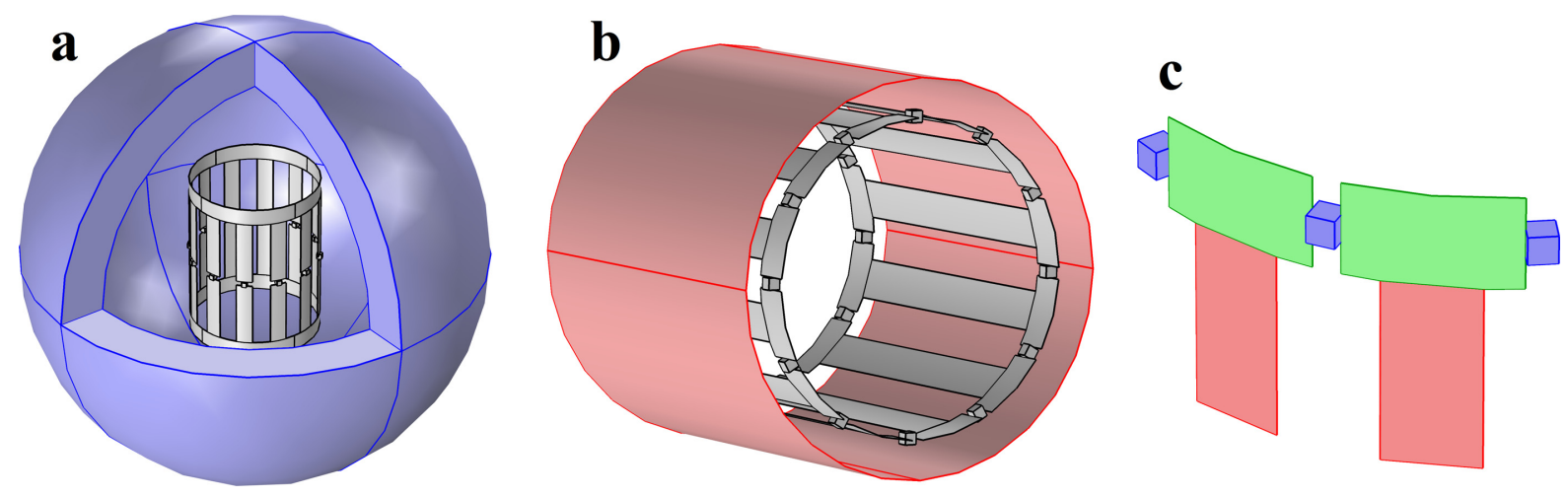

Figure 1 Geometric models of (a) 12-leg unshielded low-pass birdcage coil which is enclosed by the spherical solution domain (b) 12-leg shielded high-pass birdcage coil (c) Rungs (red), end rings (green), and capacitors (purple) of a high-pass birdcage coil.

purposes an air filled spherical finite solution domain, the radius of which is 1.5 times the maximum dimension of the birdcage coil, was created. An additional layer, the thickness of which is $25 \%$ of the radius of the solution domain, was created and the perfectly matched layer (PML) absorbing boundary condition was used for this layer to prevent reflections from the outer boundary of the solution domain [Fig. 1(a)] $(34,35)$.

For the computation of the electromagnetic field distributions, the coil is excited (in linear or quadrature mode) by a sinusoidal voltage at Larmor frequency. In linear excitation the coil is driven from one feed point, whereas in the quadrature excitation the coil is driven from two feed points that are $90^{\circ}$ apart from each other and with $90^{\circ}$ phase difference. These feed points were modeled using lumped port boundary condition (Fig. 2) (35). In COMSOL Multiphysics, use of lumped port is more appropriate than the use of port boundary condition when the mode of excitation is not known or when there is an applied voltage to the port.

As shown in Fig. 2, red surface corresponds to the lumped port boundary, green surfaces that are opposite to each other correspond to the metallic capacitor plates (only one of them is seen), and the purple volume corresponds to the capacitor domain. Lumped port boundary is used to apply a voltage between two capacitor plates and is characterized by the port voltage, $V^{+}$, (assuming $V^{-}$is ground), port current, $I$, and port impedance, $Z$. Here, $V^{+}$is the line integral of the electric field between terminals on the lumped port boundary along the width, $w$, and $I$ is defined as the integral of the surface current density, $\mathbf{J}_{\mathbf{s}}$, along the height, $h . Z$ can be found directly using the relation

$$
Z=\frac{V^{+}}{I}=\frac{\int_{w} E_{w} d l}{\int_{h} J_{s} d l} \approx \eta \frac{w}{h}
$$

where $\eta$ is the surface impedance and is found by dividing the tangential electric field to the surface current density, $\eta=\frac{E_{w}}{J_{s}}$.

In generating a mesh for the given solution domain, triangular elements were used for 2D surfaces, tetrahedral elements were used for the capacitors and air domain, and triangular prism elements were used in PML domain. The resolution and quality of the mesh elements are important quantities since low mesh element resolution may lead to inaccurate results and low mesh quality may cause

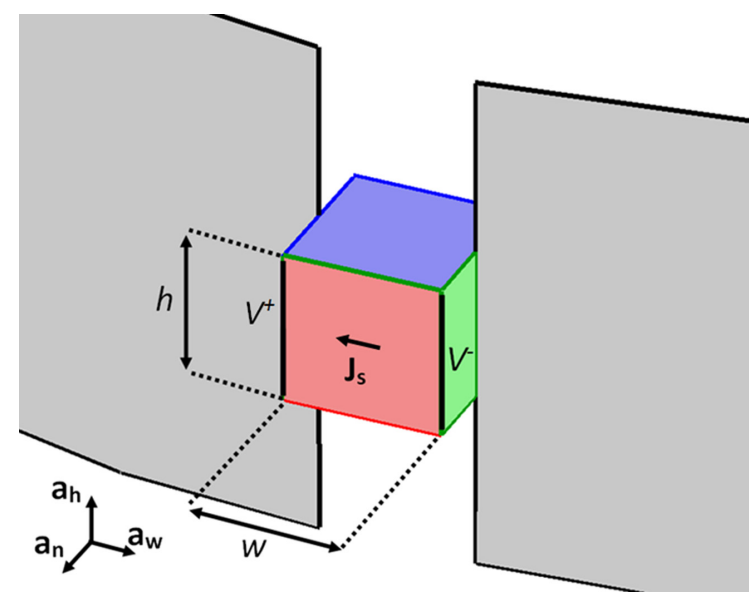

Figure 2 Illustration of a lumped port boundary (red) placed between metallic (PEC) capacitor plates (green). $h$ and $w$ are height and width of the lumped port boundary, respectively. $V+$ is the port voltage, $V-$ is the reference voltage (ground), $J_{\mathrm{s}}$ is the surface current density at the lumped port boundary. 


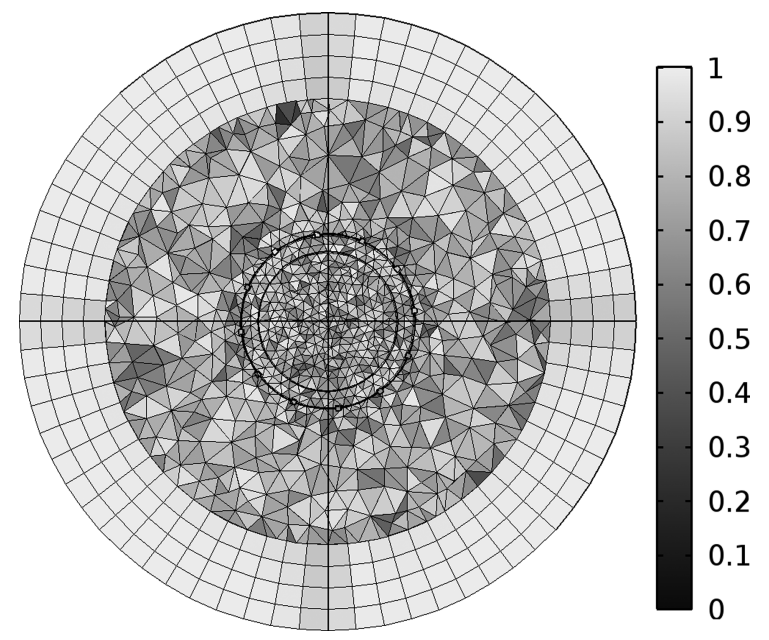

Figure 3 Mesh plot of the whole solution domain at the $x-y$ plane $(z=0$ slice). The color map shows the quality of the mesh elements. For the whole 3D solution domain, generated mesh has 226035 elements, the average mesh element quality is found as 0.74 , and the minimum mesh element quality is found as 0.13 .

convergence problems. In Comsol Multiphysics, mesh quality, which is a unitless scalar quantity (varies between 0 and 1) measures the regularity of the mesh elements' shapes (1 corresponds to a perfectly regular element, whereas 0 corresponds to a degenerated element). In Fig. 3, the generated mesh for a 12-leg high-pass birdcage coil model with a diameter of $10 \mathrm{~cm}$ and length of $12 \mathrm{~cm}$ is shown.

\section{Time-Harmonic Electromagnetic Field Solutions}

Computation of the electromagnetic field distributions inside the coil at the desired frequency is very important in terms of understanding the interactions between the RF electromagnetic fields and the object (or human subject), especially for imaging of local SAR distributions. Additionally such computations are necessary in analyzing and developing new techniques such as magnetic resonance electrical properties tomography (MREPT) (36-38) and $\mathrm{B}_{1}$ shimming. In this work, electromagnetic field calculations in the ROI were made using linear or quadrature birdcage coils. Feeding boundaries (lumped ports) of the linear and quadrature birdcage coils were modeled as lumped ports shown in Fig. 2.

It is known that for a birdcage coil, currents in the rung elements ideally must have sinusoidal current distribution in order to produce homogenous $B_{1}^{+}$magnitude inside the coil. However, it was previously shown (8) that currents do not have sinusoidal distribution because of the interactions between coil elements and the object inside the coil especially at high frequencies. In our solutions, no assumption was made for the rung elements that they have a sinusoidal current distribution to model the realistic case and the required homogeneity of the $B_{1}^{+}$magnitude is directly related to the capacitance values placed in the rungs (or/and end rings). The calculation of this capacitance value which gives the most homogenous $B_{1}^{+}$magnitude inside the unloaded birdcage coil is explained in the following section.

After geometrically modeling the birdcage coil, applying the boundary conditions and generating mesh elements for the given geometry, electromagnetic fields in the ROI at the desired frequency were computed using COMSOL Multiphysics by solving the electric field based wave equation given by

$$
(\nabla \times \nabla \times \mathbf{E})-\left(\omega^{2} \mu \varepsilon-j \omega \mu \sigma\right) \mathbf{E}=0
$$

with permeability $\mu$, permittivity $\varepsilon$, and conductivity $\sigma$.

\section{Optimum Capacitance Calculation}

In order to obtain a homogenous $B_{1}^{+}$magnitude as well as high SNR inside the unloaded birdcage coil at the desired resonance frequency, correct capacitance value must be used. In practice, therefore, after constructing the birdcage coil, tuning and matching procedures take place to make the coil resonate exactly at the desired frequency. If the initial capacitance value is very different from the necessary capacitance value, these tuning and matching procedures will be more time-consuming, and sometimes it is difficult to achieve the desired resonance pattern. In order to calculate this initial capacitance value more accurately even at high frequencies, FEM based optimization method is presented using two different objective functions: magnitude of the port impedance $(|Z|)$ and the variance of the $B_{1}^{+}$magnitude in the ROI.

We have observed that the peak values of $|Z|$ of a birdcage coil occur at the resonant modes (39). This is similar to the behavior of a parallel LC circuit at resonance. In fact, the lumped element models of both low-pass and high-pass birdcage coils can be viewed as a parallel LC circuit. $|Z|$ at the lumped port boundary of an 8-leg low-pass birdcage coil with a fixed capacitance value of $11.6 \mathrm{pF}$ is calculated by solving Eq. [1] and the variation of $|Z|$ with respect to frequency is shown in Fig. 4(a).

As can be seen in Fig. 4(a), there are four peaks that correspond to the four resonant modes of the low-pass birdcage coil. Among these resonant modes, we are interested in the resonant mode 

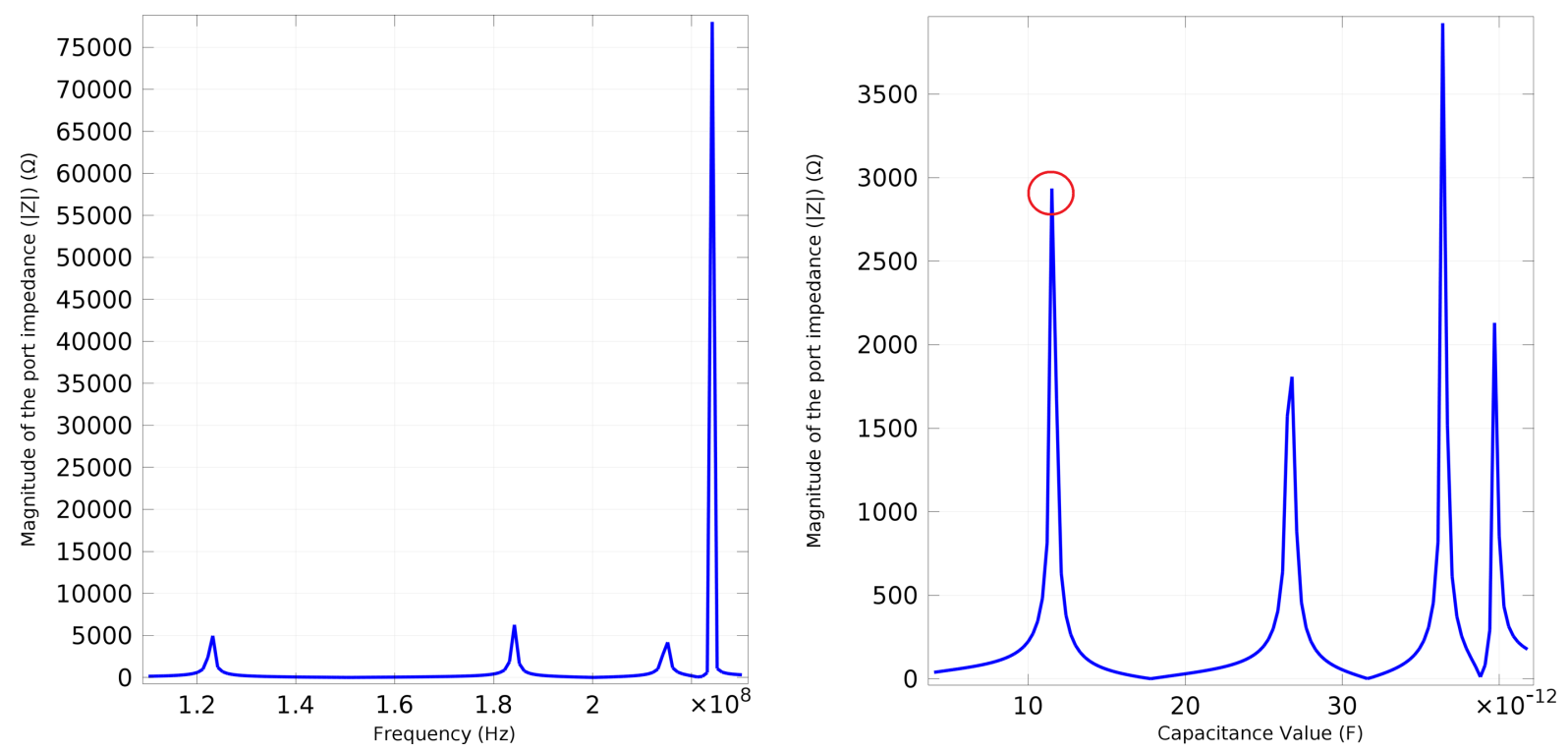

Figure $4 \mathrm{IZ}$ of an 8-leg low-pass birdcage coil with a diameter of $10 \mathrm{~cm}$, rung length of $10 \mathrm{~cm}$, and rung (and end ring) width of $1.5 \mathrm{~cm}$ (a) with respect to frequency for fixed capacitance value $(11.6 \mathrm{pF})$ and (b) with respect to capacitance for fixed frequency $(123.2 \mathrm{MHz})$.

which generates homogenous $B_{1}^{+}$magnitude inside the coil and this mode corresponds to the lowest frequency (about 123.2 MHz) in the resonance frequency spectrum for the low-pass birdcage coil. On the other hand, one can make simulations by keeping the frequency constant and varying the capacitance value. Figure 4(b) shows the dependence of IZI on the capacitance value for the fixed frequency 123.2 MHz. The peak value enclosed by the red circle in Fig. 4(b) occurs when the capacitance value is about $11.6 \mathrm{pF}$, and this peak corresponds to the first peak in Fig. 4(a). As the capacitance increases, the frequency of the first resonant mode (homogeneous mode) decreases below 123.2 MHz. The next peak in Fig. 4(b) that is seen at a capacitance value of $28 \mathrm{pF}$ corresponds to the second resonant mode of that capacitance. Similarly, the third and the fourth resonant modes for the capacitance values of $37 \mathrm{pF}$ and $40 \mathrm{pF}$ are observed in Fig. 4(b), respectively. In other words, the frequency of the first resonant mode of $11.6 \mathrm{pF}$, the frequency of the second resonant mode of $28 \mathrm{pF}$, the frequency of the third resonant mode of $37 \mathrm{pF}$, and the frequency of the fourth resonant mode of $40 \mathrm{pF}$ are all equal to $123.2 \mathrm{MHz}$. From the point of view of optimization, $|\mathrm{Z}|$ given in Fig. 4(b) is the objective function, the capacitance value is the control variable, and the task is to find the optimum capacitance value which maximizes $|Z|$ in a given capacitance range.
Alternatively, one can also use the variance of $B_{1}^{+}$magnitude as an objective function. At the resonance frequency, it is desired that the birdcage coil has a uniform $B_{1}^{+}$magnitude distribution, especially in the central region of the coil. Therefore the variance of $B_{1}^{+}$magnitude inside the unloaded birdcage coil will have its minimum value at that frequency. In order to observe this phenomenon, a square plane region was defined inside the FEM model of a birdcage coil, shown in Fig. 5(a), and Eq. [2] was solved for fixed frequency (123.2 MHz) and different capacitance values. The variance of $B_{1}^{+}$magnitude in the square shaped boundary was calculated for each capacitance value using the equation

$$
\operatorname{Var}\left(\left|B_{1}^{+}\right|\right)=\frac{1}{S_{\Omega}} \int_{\Omega}\left|B_{1}^{+}-\mu\right|^{2} d \Omega
$$

where $\Omega$ is the surface, $S_{\Omega}$ is the area of the surface, and $\mu$ is the mean of $B_{1}^{+}$in the region. The variance of $B_{1}^{+}$magnitude with respect to capacitance values is given in Fig. 5(b).

As can be seen in Fig. 5(b), $\operatorname{Var}\left(\left|B_{1}^{+}\right|\right)$has a global minimum when the capacitance value is about $11.6 \mathrm{pF}$. This minimum actually corresponds to the red circle in the $|Z|$ graph given in Fig. 4(b). From the point of view of optimization, $\operatorname{Var}\left(\left|B_{1}^{+}\right|\right)$ is the objective function, capacitance value is the control variable, and the task is to determine the optimum capacitance value which minimizes the $V a$ $r\left(\left|B_{1}^{+}\right|\right)$at the square region (or ROI). 

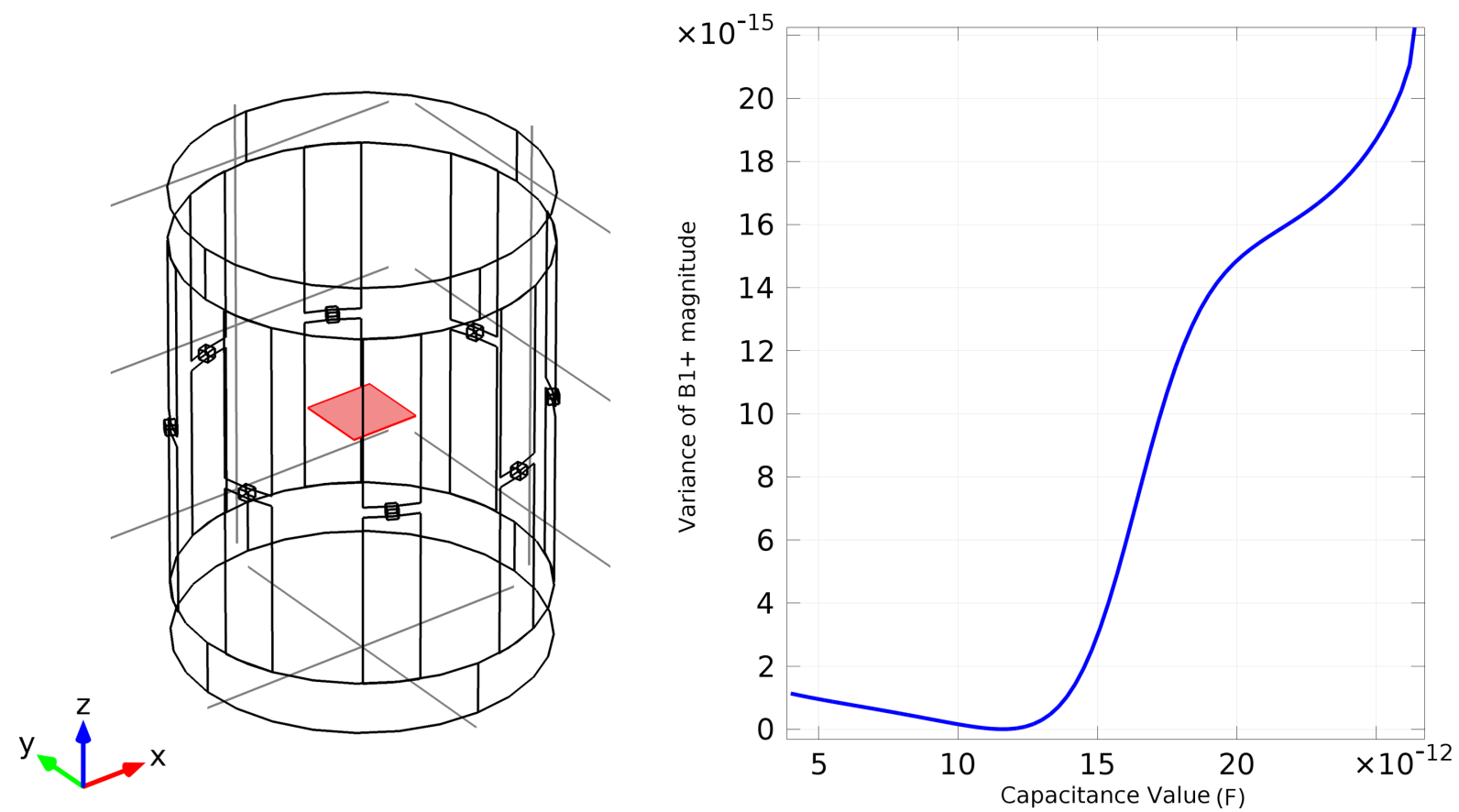

Figure 5 (a) Geometric model of an 8-leg low-pass birdcage coil with a square shaped boundary at the center of the coil (b) variance of $B_{1}^{+}$magnitude with respect to capacitance for fixed frequency $(123.2 \mathrm{MHz})$.

By investigating both objective functions, $|Z|$ and $\operatorname{Var}\left(\left|B_{1}^{+}\right|\right)$, it is apparent that $|Z|$ makes sharp peaks, whereas $\operatorname{Var}\left(\left|B_{1}^{+}\right|\right)$forms a shallow minimum. Therefore using $|Z|$ as an objective function seems more appropriate than using the $\operatorname{Var}\left(\left|B_{1}^{+}\right|\right)$ because the minimum of a shallow region cannot be found accurately due to the numerical errors in the computations. However, using only $\mid \mathrm{Zl}$ as an objective function may also give an unreliable result by converging to a local minimum if $|Z|$ has more than one peak for the specified capacitance range where optimization is made. Therefore, in order to find the optimum capacitance value, a "reasonable" capacitance range is defined by looking at $\operatorname{Var}\left(\left|B_{1}^{+}\right|\right)$ since its global minimum occurs around the desired capacitance range and optimization is made using $|Z|$ as an objective function in this capacitance range. The step by step procedure for determination of the "reasonable" capacitance range and the optimum capacitance value is explained in the simulation tools section.

\section{Resonant Mode Analysis}

A birdcage coil with number of legs, $N$, and equal valued capacitors has $N / 2$ (or $N / 2+1$ ) resonant modes among which only one mode has the desired homoge- nous magnetic field distribution. For a low-pass birdcage coil, the lowest resonance frequency in the resonance frequency spectrum corresponds to this homogenous mode. For a high-pass birdcage coil, on the other hand, the highest resonance frequency (excluding the highest frequencies corresponding to the anti-rotating and co-rotating ring modes in which the currents flow only in the end rings (3)) corresponds to this desired homogenous mode. To calculate the resonant modes of a birdcage coil directly, without making any frequency sweep, this study presents the resonant mode analysis of a birdcage coil using the eigenvalue solver of COMSOL Multiphysics.

In the case of an eigenvalue problem, no source (or excitation) is applied to the model. Therefore, the same geometry explained in the FEM models of birdcage coils section was built but no lumped port boundary condition was used. The governing equation is the same as Eq. [2], but $\omega$ is the unknown parameter and the eigenvalue $(\lambda)$, which is to be solved, can be expressed in terms of $\omega$ as

$$
-\lambda=-\delta+j \omega
$$

where the imaginary part of the eigenvalue $(\omega)$ corresponds to the undamped eigenfrequency and the real part $(\delta)$ represents the damping factor. In our case, using perfectly matched layer as an outer 


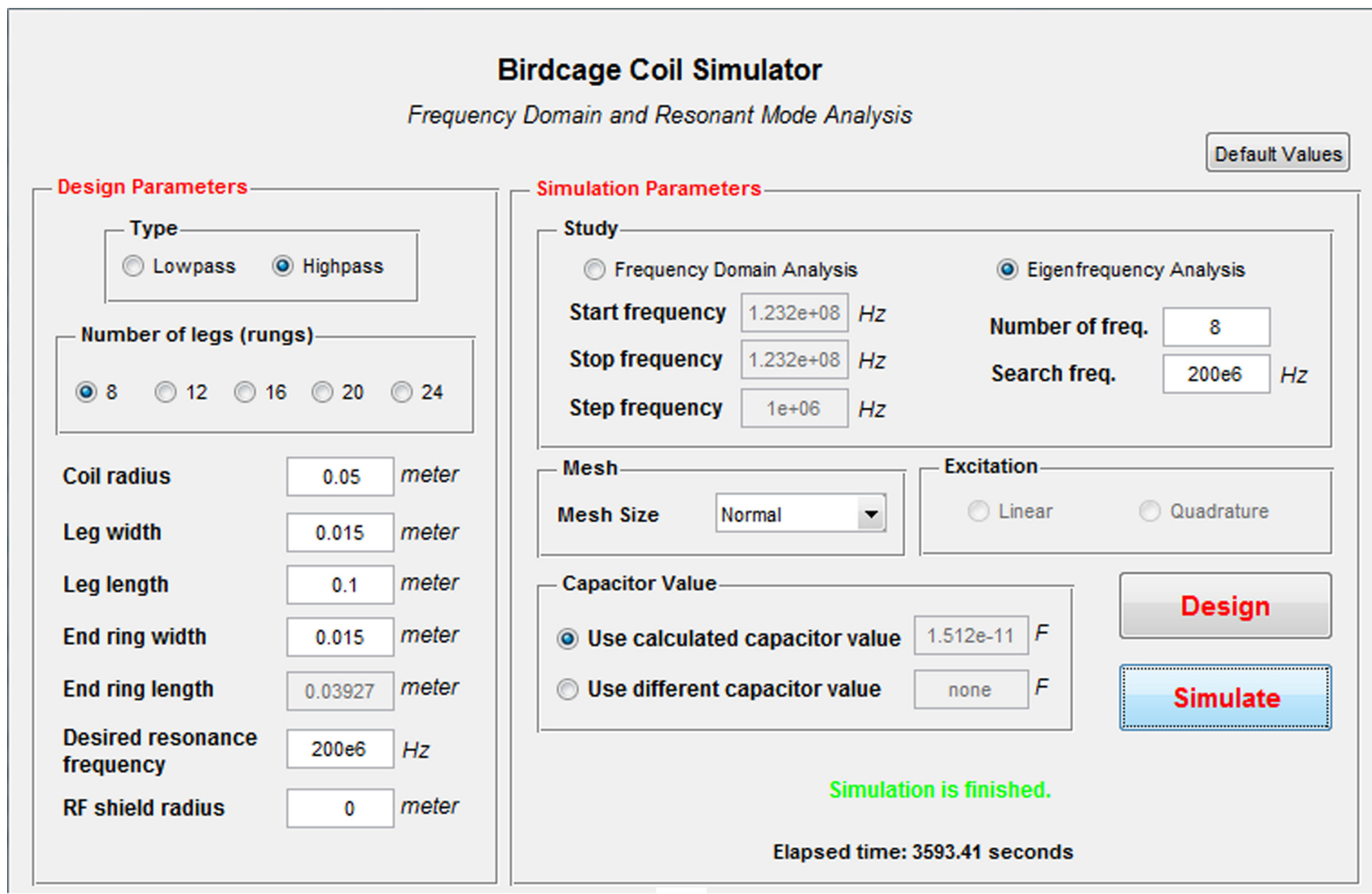

(a)

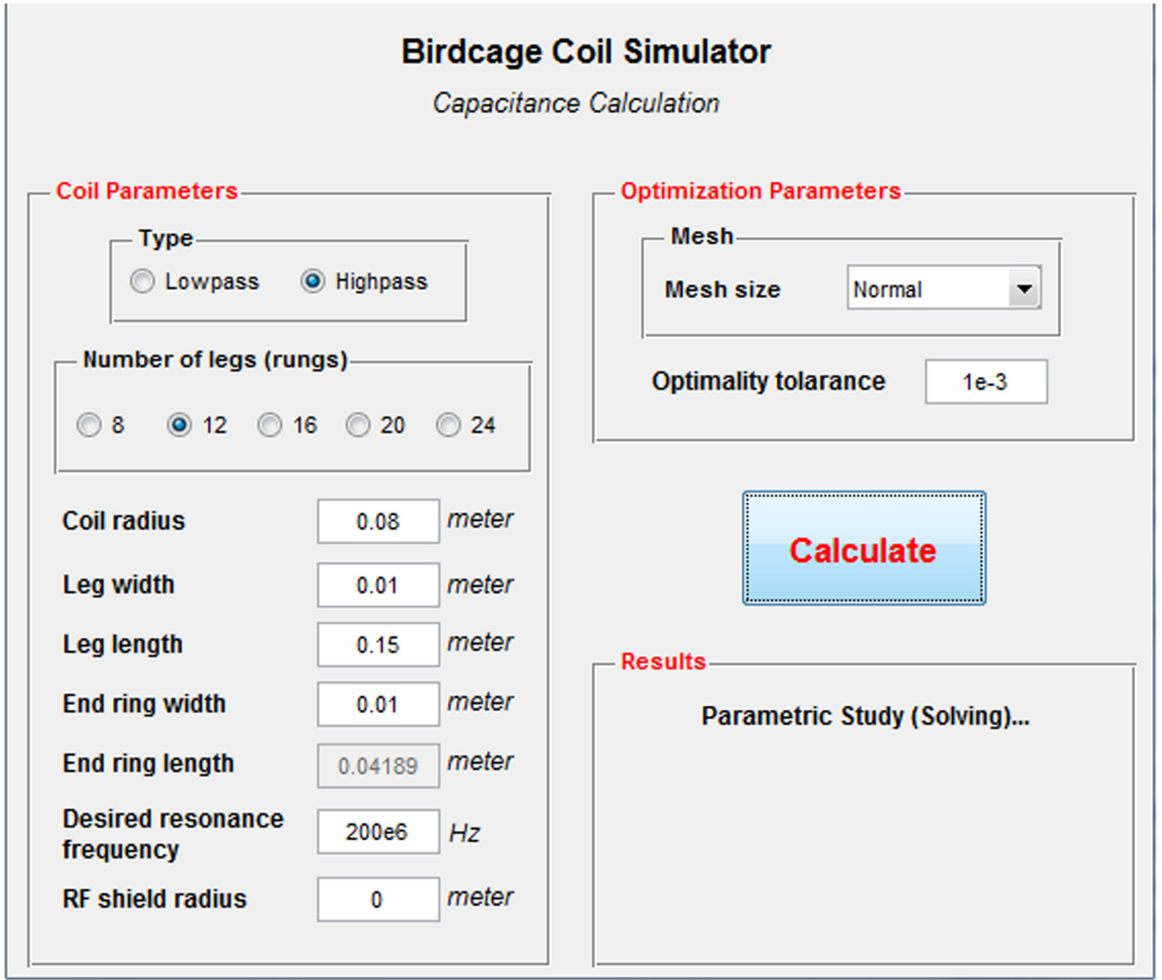

(b)

Figure 6 Graphical user interfaces of the simulation tools: (a) for frequency domain and resonant mode analysis (RM-FDA) (b) for optimum capacitance calculation (OptiCF). 
boundary condition introduces a loss in the model and therefore the damped eigenfrequency must be considered, and this can be calculated as

$$
\omega_{d}=\omega \sqrt{1-\zeta^{2}}
$$

where $\zeta$ is the damping ratio and is expressed as

$$
\zeta=\frac{\delta}{\omega}
$$

\section{Software Tools}

In order for the proposed methods to be conveniently applicable, two software tools, which also have user graphical interfaces (GUIs), were developed using MATLAB. Through LiveLink ${ }^{\mathrm{TM}}$ for MATLAB, which is the interface between COMSOL Multiphysics and MATLAB, FEM modeling functionalities of COMSOL Multiphysics; i.e., modeling of the geometry, adding physics and boundary conditions, making discretization (mesh generation) and computing the solutions, are used in MATLAB. (These software packages and their source codes are available for free at http://www.ee.bilkent.edu.tr/ emtpbiomed/bcs-request.html)

One of the simulation tools, the GUI of which is shown in Fig. 6(a), is used to compute the electromagnetic fields or the resonant modes of a birdcage coil for the desired design and simulation parameters. This tool is called the resonant mode and frequency domain analyzer (RM-FDA).

As can be seen in Fig. 6(a), the user should specify the Design Parameters related to the coil type and geometry, and the Simulation Parameters related to the study type, mesh size, and excitation type. If frequency domain analysis is chosen as a study type, the desired frequency range and the excitation type (linear or quadrature) must be specified. On the other hand, if eigenfrequency analysis is chosen, the user should specify the number of modes to be found, and the single frequency around which the solver finds the resonant modes of a birdcage coil (The eigenvalue problem considered in this study is nonlinear, and therefore the problem is linearized around the given frequency). In addition to specifying all Design and Simulation parameters, the user should also specify the value of the capacitance in the rungs (or end rings). This capacitance value can either be calculated using the lumped circuit element modeling method (6) by clicking the Design button or can be specified directly by the user. After specifying the capacitance value to be used, the desired simulation study is started by clicking the Simulate button. When a simulation is finished, the user can investigate the solutions in either COMSOL Multiphysics or MATLAB environment.

The other software tool, which is called the Optimum Capacitance Finder (OptiCF), is used to calculate the optimum capacitance value of a birdcage coil to resonate the coil at the specified frequency. The GUI of OptiCF is shown in Fig. 6(b). By clicking the Calculate button, the software tool first calculates an approximate capacitance value $\left(C_{\mathrm{lp}}\right)$ for the given parameters using lumped circuit modeling method (6). An approximate capacitance range is then defined around this capacitance value as $\left[C_{\min }, C_{\max }\right]$ where $C_{\min }$ and $C_{\max }$ are in $\mathrm{pF}$ and

$$
\begin{aligned}
& {\left[C_{\min }, C_{\max }\right]=} \\
& \left\{\begin{array}{l}
{\left[\left(0.9 C_{l p}\right),\left(1.1 C_{l p}\right)\right] \text { for } \alpha<1} \\
{\left[\left(C_{l p}-\alpha\right),\left(C_{l p}+\alpha\right)\right] \text { for } \alpha>1}
\end{array}\right.
\end{aligned}
$$

where $\alpha$ is determined empirically using the maximum dimension of the coil and the wavelength $(\lambda)$ as

$$
\alpha=\frac{\max (D, L)}{\lambda / 20}
$$

where $D$ is the diameter of the coil, and $L$ is the rung length of the coil. The idea behind this capacitance range definition is that as the frequency increases to a point where the coil dimensions become comparable with one twentieth of the wavelength, lumped circuit element modeling method yields more error, and therefore $\alpha$ is increased in order not to miss the capacitance value corresponding to the homogenous mode.

After defining the approximate capacitance range $\left[C_{\min }, C_{\max }\right]$, a linearly driven unloaded birdcage coil is modeled with the "very coarse" mesh of COMSOL Multiphysics and the electromagnetic fields are computed for different capacitance values in this capacitance range. As a result of this parametric sweep study, two objective functions, $|\mathrm{Z}|$ and $\operatorname{Var}\left(\left|B_{1}^{+}\right|\right)$with respect to the capacitance values are calculated and displayed to the user. By investigating the graphs of these two objective functions, a narrower and "reasonable" capacitance range in the $|Z|$ graph, where the optimization is to be made, is selected. Here the "reasonable" capacitance range implies that $|Z|$ has only one maximum point in this range. This procedure is explained with the following example: for a 12-leg high-pass birdcage coil with a diameter of $16 \mathrm{~cm}$, rung length of $15 \mathrm{~cm}$ and the desired frequency of 200 

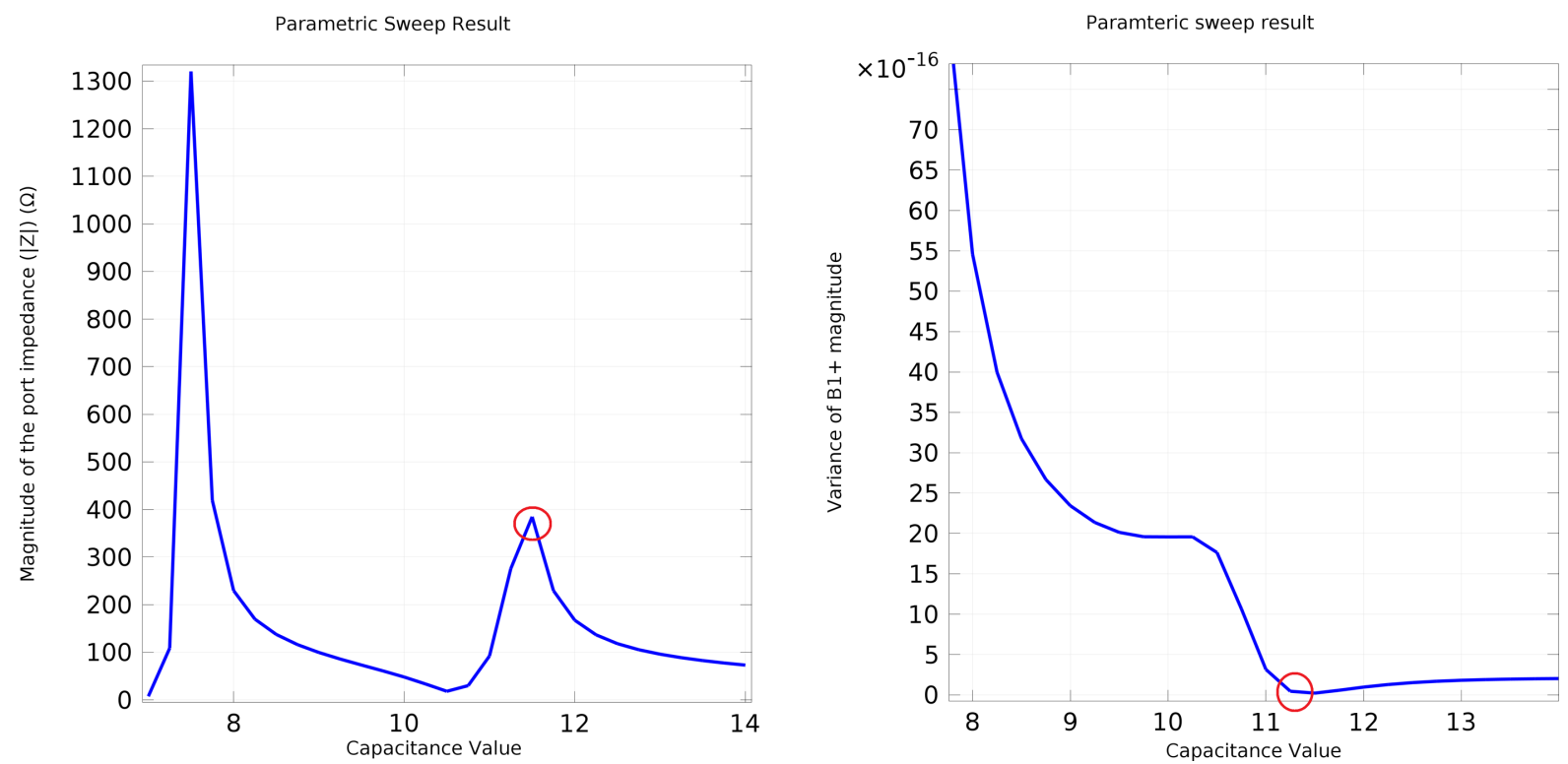

Figure 7 Parametric sweep results for the 12-leg unshielded high-pass birdcage coil with a diameter of $16 \mathrm{~cm}$, rung length of $15 \mathrm{~cm}$, rung (and end ring) width of $1 \mathrm{~cm}$ at $200 \mathrm{MHz}$. (a) $\mathrm{IZ|}$ in the approximate capacitance range (b) $\operatorname{Var}\left(\left|B_{1}^{+}\right|\right)$in the approximate capacitance range (capacitance values are in $\mathrm{pF}$ ).

$\mathrm{MHz}, \mathrm{IZ|}$ and $\operatorname{Var}\left(\left|B_{1}^{+}\right|\right)$are calculated with respect to capacitance values (in the defined approximate capacitance range) and are shown in Figs. 7(a,b), respectively.

As can be seen in Fig. 7(a), IZI has more than one peak in the determined approximate capacitance range but we are interested in the peak of $|Z|$ which corresponds to the global minimum of $\operatorname{Var}\left(\left|B_{1}^{+}\right|\right)$. This peak and the corresponding global minimum are encircled in Figs. 7(a,b). The user is then requested to select the "reasonable" capacitance range around this peak by clicking three points on the $|Z|$ graph. These three points correspond to the lower bound, initial value and the upper bound of the control variable (capacitance value) which are to be used in the optimization process. After specifying the final capacitance range for the optimization, the software tool starts the optimization process with a "very fine" mesh of COMSOL Multiphysics.

\section{Simulation Methods}

Simulations were performed for both unloaded and loaded birdcage coils using the developed simulation tools, OptiCF and RM-FDA. The machine used for the simulations is HP Z800 workstation with Intel Zeon X5675 $3.07 \mathrm{GHz}$ dual processors (12 cores) and with 64 GB RAM. For the unloaded case, different sizes and types of birdcage coils were modeled; resonant modes and time-harmonic electromagnetic fields of these birdcage coils were analyzed in order to validate the developed software tools. For the loaded case, on the other hand, 16-leg shielded birdcage head coil was modeled and loaded with the human head model as shown in Fig. 8(a).

The human head was simply modeled as consisting of five tissues: scalp, skull, cerebrospinal fluid (CSF), white matter (WM), and grey matter (GM). For this purpose, volumetric segmented MRI images $\left(215 \times 180 \times 70,1 \times 1 \times 2.5 \mathrm{~mm}^{3}\right)$ obtained from $(40,41)$ were used. Although these segmented images consist of 11 different tissue classes, they were merged and formed as the aforementioned five tissues. In order to export the geometry of the human head model to COMSOL Multiphysics, mesh generation toolbox, iso2mesh $(42,43)$, was used to mesh the volumetric segmented MRI images and meshed geometry was saved as STereoLithography (STL) format which can be imported by COMSOL Multiphysics. Finally, material properties (conductivity and relative permittivity values) given in Table 1 were used for the human head model. The conductivity and permittivity images for different planes are shown in Figs. 8(b,c). Using this head model, distributions of the electromagnetic fields at $3 \mathrm{~T}$ and $7 \mathrm{~T}$ and the normalized SAR values were computed. Furthermore, frequency shifts in 

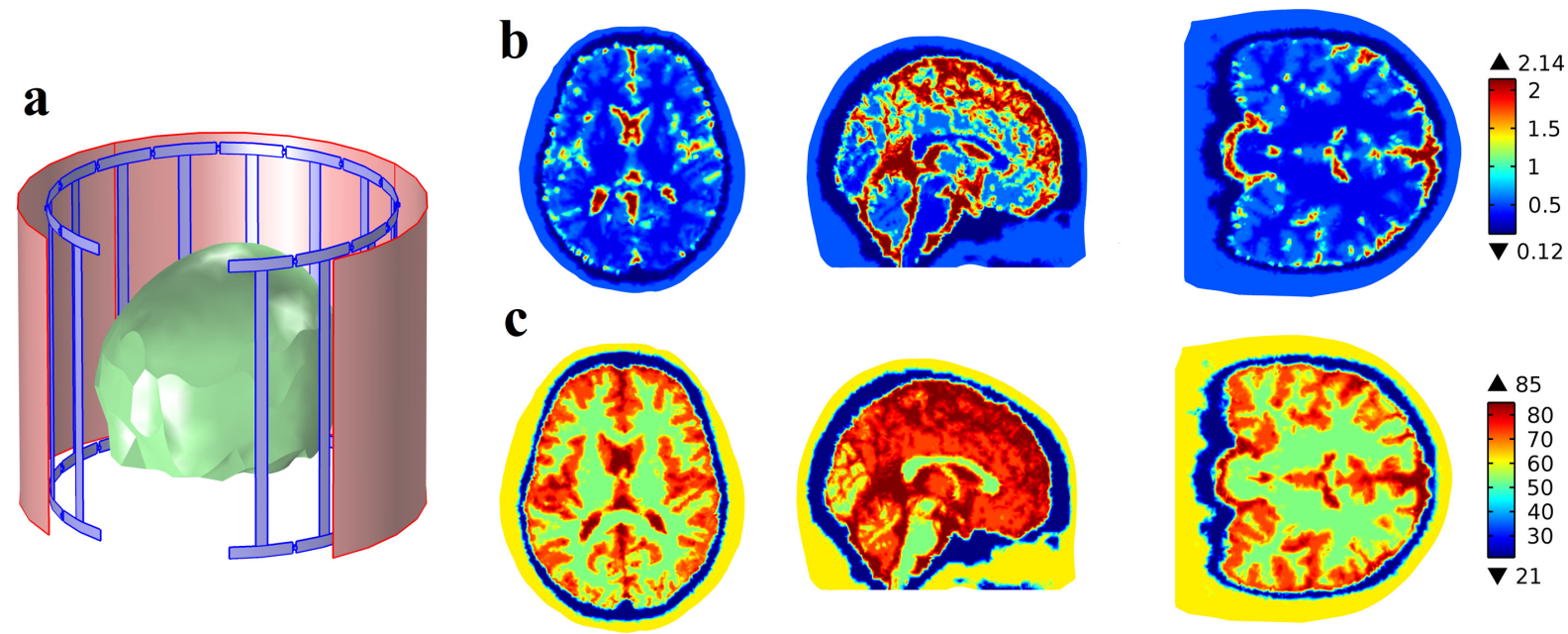

Figure 8 (a) Human head model inside the 16-leg head birdcage coil (b) Interpolated conductivity images for transverse (left), sagital (mid), and coronal plane (right) (c) Interpolated permittivity images for transverse (left), sagital (mid), and coronal plane (right) for $128 \mathrm{MHz}$.

the resonant modes of the loaded birdcage coils were calculated by making resonant mode analysis.

\section{Experimental Methods}

To verify the results obtained by the proposed numerical methods regarding capacitance values and resonant mode frequencies, experimental measurements were also performed. For this purpose, two handmade birdcage coils (low-pass and highpass) were constructed on plexiglass tubes using adhesive copper strips and they are illustrated in Fig. 9.

Resonant mode frequencies of each birdcage coil were measured for five different capacitance values (Dielectric Laboratories High-Q Multi-Layer and Broadband Blocking Capacitors) by observing the $\mathrm{S}_{11}$ seen from the feed point of the coil using Agilent Technologies E5061A Network Analyzer. These measured resonant modes were compared

Table 1 Material Properties of Five Tissues of the Head Model

\begin{tabular}{lccccc}
\hline & \multicolumn{2}{c}{$128 \mathrm{MHz}$} & & \multicolumn{2}{c}{$300 \mathrm{MHz}$} \\
\cline { 2 - 3 } \cline { 6 - 6 } Tissue & $\sigma(\mathrm{S} / \mathrm{m})$ & $\varepsilon_{r}$ & & $\sigma(\mathrm{S} / \mathrm{m})$ & $\varepsilon_{r}$ \\
\hline CSF & 2.14 & 84 & & 2.22 & 73 \\
WM & 0.34 & 52 & & 0.41 & 44 \\
GM & 0.59 & 73 & & 0.69 & 60 \\
Skull & 0.12 & 21 & & 0.14 & 18 \\
Scalp & 0.54 & 62 & & 0.64 & 50 \\
\hline
\end{tabular}

with the resonant modes calculated using the RMFDA.

For each capacitance value, $C_{\mathrm{e}}$, used in the resonant mode frequency measurement, the homogenous mode frequency was noted. This frequency is in turn used as the "desired resonance frequency" input of OptiCF in order to find an optimum capacitance value, $C_{\mathrm{opt}}$. In addition to OptiCF, the same desired resonance frequency was specified as input to the BirdcageBuilder software tool (6) to find the necessary capacitance value, $C_{\mathrm{BB}}$. These calculated $C_{\text {opt }}$ and $C_{\mathrm{BB}}$ values were then compared with the experimentally used capacitance value, $C_{\mathrm{e}}$, in order to assess the relative accuracies of our proposed software tool, OptiCF, and the lumped circuit element model based software tool, BirdcageBuilder.

\section{RESULTS}

\section{Simulation Results}

Unloaded Case-Validation and Verification of the Software Tools. Although simulations for unloaded birdcage coils are basic and the results are known, we have nevertheless made use of unloaded case simulations to test the proposed software tools. One of the simulations was made for unloaded and unshielded 12-leg high-pass birdcage coil with a diameter of $16 \mathrm{~cm}$ and a length of $16 \mathrm{~cm}$ at 200 MHz. Using OptiCF, the optimum capacitance value to make the coil resonate at this frequency was found as $11.15 \mathrm{pF}$. Specifying this optimum 

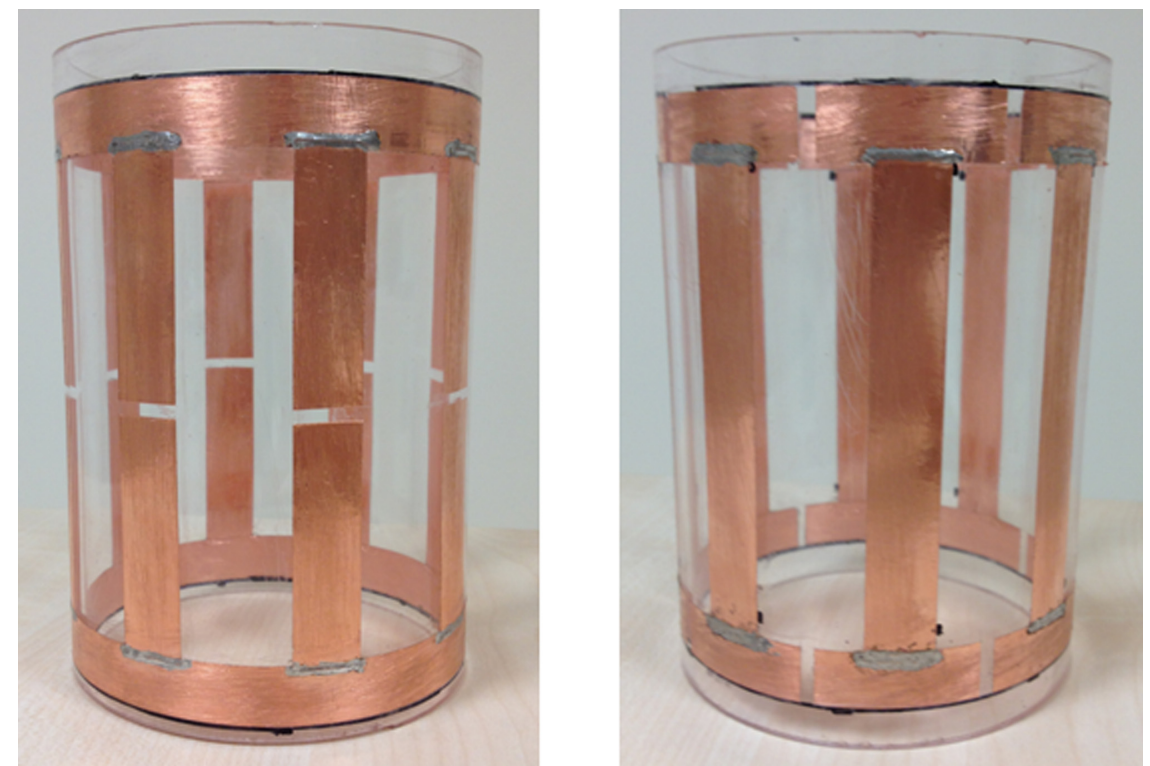

Figure 9 Constructed handmade birdcage coils with a diameter of $10 \mathrm{~cm}$, a rung length of $11.5 \mathrm{~cm}$. The width of the copper strips used to construct rungs and end rings is $1.5 \mathrm{~cm}$. Low-pass birdcage coil without capacitors (left) and high-pass birdcage coil without capacitors (right).

capacitance value in the RM-FDA, resonant mode analysis of this birdcage coil was performed. $\left|B_{1}^{+}\right|$ images for calculated six different resonant modes are given in Fig. 10. Each of these six different resonant modes in fact has duplicate eigenvalues due to fourfold symmetry of a 12-leg birdcage coil (1).

When the optimum capacitance value was used in the resonant mode analysis, it was found that homogenous mode [Fig. 10(a)] is at $199.71 \mathrm{MHz}$ which is very close to the desired resonance frequency, and this shows that developed software tools, RM-FDA and OptiCF, are consistent with each other. As can be seen in Figs. 10(b-f), for the other resonant modes, $\left|B_{1}^{+}\right|$distributions are not homogenous, and furthermore they have low magnitude. Therefore the mode at $199.71 \mathrm{MHz}$ is the one to be used in MR applications.

For the second case, 8-leg shielded and unloaded low-pass birdcage coil with a diameter of $10 \mathrm{~cm}$ and length of $11 \mathrm{~cm}$ were modeled and the optimum capacitance value, which is necessary to resonate the coil at $298.2 \mathrm{MHz}$, was calculated using OptiCF. This optimum capacitance value $(1.62 \mathrm{pF})$ and the desired resonance frequency $(298.2 \mathrm{MHz})$ were then used in RM-FDA and time-harmonic electromagnetic fields of the coil were computed for both linear and quadrature excitations. $\left|B_{1}^{+}\right|,\left|B_{1}^{-}\right|$ (left-hand rotating and right-hand rotating components of the magnetic field respectively), and mag- nitude of the electric field $(|E|)$ were investigated for each excitation, and the results are illustrated in Fig. 11.

As can be seen in Figs. 11(e-g), $\left|B_{1}^{+}\right|$and $\left|B_{1}^{-}\right|$ have almost same distributions in linear excitation case and their combination produces a linearly polarized field inside the coil. When the same birdcage coil is driven in a quadrature mode, $\left|B_{1}^{+}\right| \gg \mid$ $B_{1}^{-} \mid$and $\left|B_{1}^{-}\right|$is nearly zero in the central region of the coil [Figs. 11(a-c)] and therefore circularly polarized field due to the $\left|B_{1}^{+}\right|$is generated inside the coil. IEl-field images for linear and quadrature excitation cases are also shown in Figs. 11(d,h), respectively.

Loaded Case-Application of the Software Tools. After validation of the proposed software tools, more complex simulations were performed by loading the 16-leg shielded birdcage head coil with a human head model as shown in Fig. 8(a). The coil has a diameter of $29 \mathrm{~cm}$, a shield diameter of $34 \mathrm{~cm}$, and a rung length of $24 \mathrm{~cm}$. The widths of the rungs and end rings were chosen as $1 \mathrm{~cm}$. The optimum capacitance value for the given desired resonance frequency, $128 \mathrm{MHz}(\sim 3 \mathrm{~T})$ and 300 $\mathrm{MHz}(\sim 7 \mathrm{~T})$, were calculated as $32.3 \mathrm{pF}$ and $4.6 \mathrm{pF}$, respectively. Similar to unloaded case, resonant mode and time-harmonic electromagnetic analyses of the birdcage coil were made for the loaded case. 

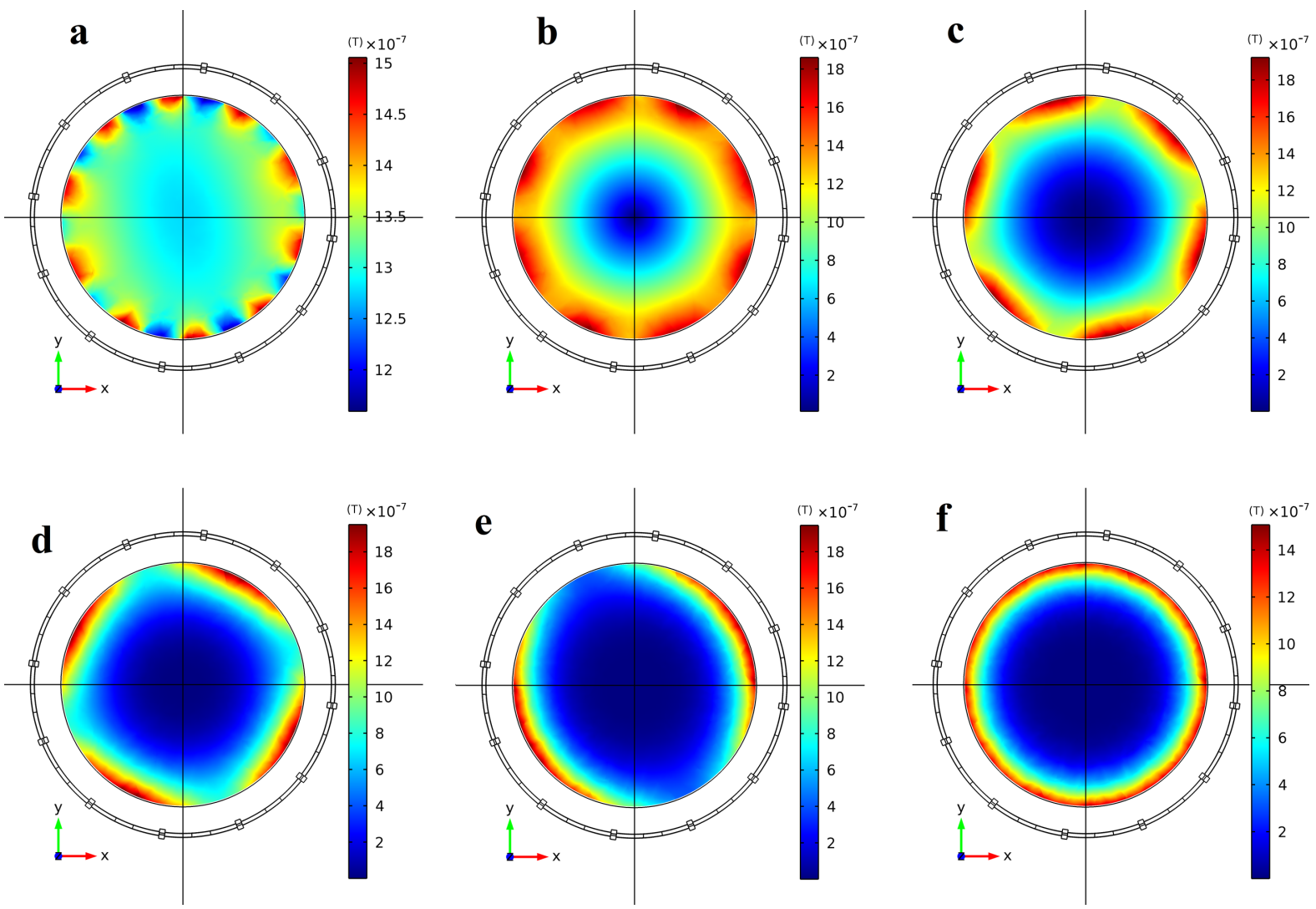

Figure $10\left|B_{1}^{+}\right|$images at $x-y$ plane ( $z=0$ slice) for each resonant mode of 12-leg high-pass birdcage coil with a diameter of $16 \mathrm{~cm}$, a rung length of $16 \mathrm{~cm}$, rung (and end ring) width of $1 \mathrm{~cm}$, and the capacitance value of $11.15 \mathrm{pF}$ : (a) the homogenous mode found at 199.71 $\mathrm{MHz}$, (b) the $2^{\text {nd }}$ resonant mode at $164.12 \mathrm{MHz}$, (c) the $3^{\text {rd }}$ resonant mode at $141.61 \mathrm{MHz}$, (d) the $4^{\text {th }}$ resonant mode at $128.35 \mathrm{MHz}$, (e) the $5^{\text {th }}$ resonant mode at $121.29 \mathrm{MHz}$, (f) the $6^{\text {th }}$ resonant mode at $119.05 \mathrm{MHz}$ (Since the magnitude of the fields near the rungs are very high, the fields are drawn in region with a diameter of $12.8 \mathrm{~cm}$ ).

Resonant mode analysis of the loaded birdcage coil at $3 \mathrm{~T}$ and $7 \mathrm{~T}$. Using RM-FDA, resonant mode analysis was made for both unloaded and the loaded birdcage coil at 3T and 7T. Calculated resonant modes are given in Table 2. For the homogenous mode, at $3 \mathrm{~T}$, the resonance frequencies for the unloaded and loaded cases are slightly different $(0.1 \%)$. At $7 \mathrm{~T}$, on the other hand, homogenous mode resonant frequency of the loaded case is higher by $1.8 \%$ than the unloaded case. Thus, it is observed that effect of loading on the resonant frequency is more significant at higher static magnetic field strengths. These results are also consistent with the literature findings but in this study these resonant modes were directly calculated (without making frequency sweep study) by making eigenfrequency analysis. This is important in terms of duration of the computations. Eigenfrequency analysis takes 15-18 minutes to compute all the resonant modes. If one uses the fre- quency sweep analysis to calculate the resonant modes, (i.e. calculating the time-harmonic electromagnetic fields for the specified frequencies [as shown in Fig. 4(a)], it will take a huge amount of time. For example, the number of mesh elements of the birdcage coil, for which the frequency sweep result is given in Fig. 4(a), is 42485. Calculating the electromagnetic fields at only one frequency for this coil takes about 2 minutes. To cover all resonant modes of the coil in the frequency range $120 \mathrm{MHz}$ to $280 \mathrm{MHz}$ and using a step frequency of $1 \mathrm{MHz}$, one needs about 5 hours. In fact in order to find the resonance frequencies with more precision one needs to use a significantly lower step frequency in which case the duration of the computations will be unacceptable. In other words, sweep based resonant mode analysis is not practical, whereas finding the resonant frequencies using eigenfrequency analysis is fast and precise. 

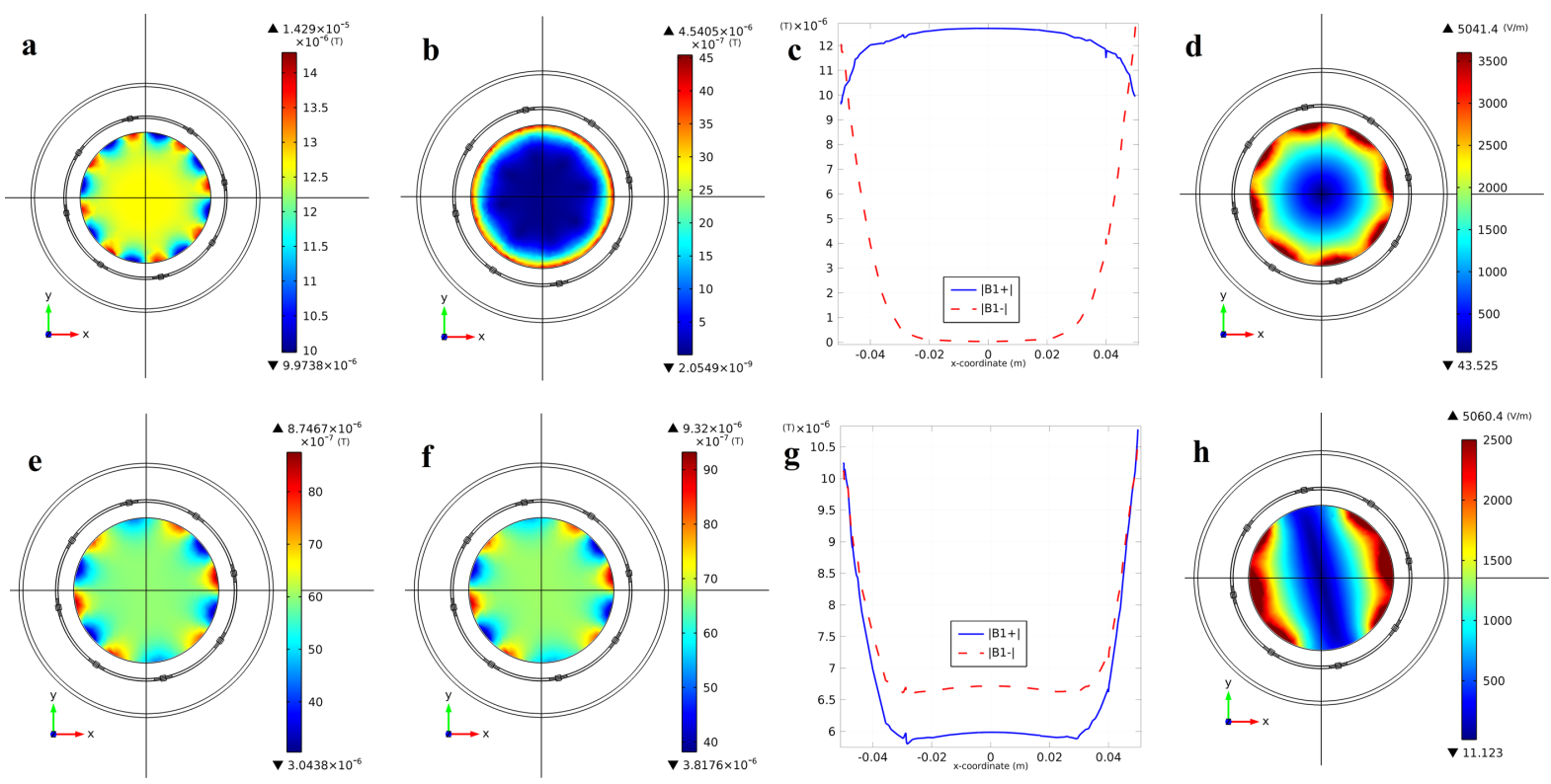

Figure $11\left|B_{1}^{+}\right|,\left|B_{1}^{-}\right|$, and $\mathrm{IEl}$ images at $x-y$ plane $(z=0$ slice) for quadrature and linear drive shielded low-pass birdcage coil with a coil diameter of $10 \mathrm{~cm}$, shield diameter of $14 \mathrm{~cm}$, rung length of $11 \mathrm{~cm}$, rung (and end ring) width of $1 \mathrm{~cm}$, capacitance value of 1.62 $\mathrm{pF}$ and the frequency of 298.2 MHz. For quadrature excitation: (a) $\left|B_{1}^{+}\right|$(b) $\left|B_{1}^{-}\right|$(c) $\left|B_{1}^{+}\right|$and $\left|B_{1}^{-}\right|$distributions along the $(x, y=0, z=0)$ line (d) IEl-field. For linear excitation: (e) $\left|B_{1}^{+}\right|$(f) $\left|B_{1}^{-}\right|(\mathrm{g})\left|B_{1}^{+}\right|$and $\left|B_{1}^{-}\right|$distributions along the $(x, y=0, z=0)$ line (h) $\mid$ El-field. (Since the magnitude of the fields near the rungs is very high, the fields are drawn in a region with diameter of $8 \mathrm{~cm}$ ).

Time-harmonic electromagnetic analysis of loaded birdcage coil at $3 \mathrm{~T}$ and 7T. Using RMFDA, time-harmonic electromagnetic fields of the loaded birdcage coil were computed both at $3 \mathrm{~T}$ and $7 \mathrm{~T}$. The coil was driven in quadrature mode. $\left|B_{1}^{+}\right|$ and $\left|B_{1}^{-}\right|$images at the central slice are shown in Fig. 12. The homogeneity of $\left|B_{1}^{+}\right|$in both cases deteriorates significantly due to the presence of the human head. At 3T, the variation of $\left|B_{1}^{+}\right|$is about $\pm 20 \%$ within a square region of $20 \mathrm{~cm}$ side length whereas at $7 \mathrm{~T}$ this variation is observed as $\pm 50 \%$. Furthermore, $\left|B_{1}^{-}\right|$is no longer close to zero when compared to unloaded case [shown in Fig. 11(b)]. Additionally central brightening at $7 \mathrm{~T}$ is more pronounced than the central brightening at 3T.

In addition to electromagnetic fields, for this case, one may also calculate the SAR value at any point $\mathbf{r}=(x, y, z)$ via

$$
\operatorname{SAR}(\mathbf{r})=\frac{\sigma(\mathbf{r})}{2 \rho(\mathbf{r})}|\mathbf{E}|^{2}
$$

where $\sigma(\mathbf{r})$ and $\rho(\mathbf{r})$ are the electrical conductivity and density of the object at point $\mathbf{r}$, respectively, and $\mathbf{E}$ is the electric field vector. For both field strengths, the magnitude of the electric field $(|\mathbf{E}|)$ and normalized SAR distributions at the central slice are shown in Fig. 13. We observe that, at 3T, SAR is low in the central region of the human head and is higher in the CSF regions adjacent to the skull. At 7T, on the other hand, SAR value is higher in the central region especially at CSF regions. The reason why SAR is low in the central CSF region at $3 \mathrm{~T}$ is that in this region electric field magnitude is low. However, at 7T, magnitude of the electric field is higher in this region when compared to the low electric field region at $3 \mathrm{~T}$ and therefore SAR is higher in the central CSF regions at $7 \mathrm{~T}$. These examples show that visualization of SAR, electric field, and magnetic field give valuable insights regarding the interactions of these variables especially at high-field strengths where these interpretations are not easy to predict without making electromagnetic calculations.

\section{Experimental Results}

Capacitance values calculated using the OptiCF and BirdcageBuilder, $C_{\mathrm{opt}}$ and $C_{\mathrm{BB}}$, were compared with the experimentally used capacitance values, $C_{\exp }$, and these capacitance values are given in Tables 3 
Table 2 Frequencies of the Resonant Modes of Loaded and Unloaded Birdcage Head Coils for Two Different Capacitance Values That Correspond to the 3T and 7T Field Strengths

\begin{tabular}{lcccccc}
\hline & \multicolumn{3}{c}{$32.3 \mathrm{pF}(\sim 3 \mathrm{~T})$} & & \multicolumn{3}{c}{$4.6 \mathrm{pF}(\sim 7 \mathrm{~T})$} \\
\cline { 2 - 6 } Modes & $\begin{array}{c}\text { Unloaded } \\
(\mathrm{MHz})\end{array}$ & $\begin{array}{c}\text { Loaded } \\
(\mathrm{MHz})\end{array}$ & $\begin{array}{c}\text { Shift } \\
(\%)\end{array}$ & $\begin{array}{c}\text { Unloaded } \\
(\mathrm{MHz})\end{array}$ & $\begin{array}{c}\text { Loaded } \\
(\mathrm{MHz})\end{array}$ & $\begin{array}{c}\text { Shift } \\
(\%)\end{array}$ \\
\hline 1st (homogenous & 127.71 & 127.58 & $\mathbf{0 . 1}$ & 299.48 & 304.80 & $\mathbf{1 . 8}$ \\
$\quad$ mode) & 101.32 & 101.26 & $\mathbf{0 . 0 6}$ & 242.73 & 242.24 & $\mathbf{0 . 2}$ \\
2nd & 82.86 & 82.84 & $\mathbf{0 . 0 2}$ & 202.58 & 202.41 & $\mathbf{0 . 1}$ \\
3rd & 71.12 & 71.12 & $\mathbf{0}$ & 176.42 & 176.40 & $\mathbf{0 . 0 1}$ \\
4th & 63.71 & 63.71 & $\mathbf{0}$ & 159.51 & 159.54 & $\mathbf{0 . 0 2}$ \\
5th & 59.21 & 59.21 & $\mathbf{0}$ & 149.05 & 149.04 & $\mathbf{0}$ \\
6th & 56.74 & 56.74 & $\mathbf{0}$ & 143.27 & 143.31 & $\mathbf{0 . 0 3}$ \\
7th & 55.97 & 55.97 & $\mathbf{0}$ & 141.46 & 141.49 & $\mathbf{0 . 0 2}$ \\
8th & 151.21 & 151.18 & $\mathbf{0 . 0 2}$ & 389.62 & 390.26 & $\mathbf{0 . 1 6}$ \\
0th (end-ring & & & & & & \\
resonant mode) & & & & & & \\
\hline
\end{tabular}

and 4 for the low-pass and high-pass birdcage coils, respectively.

Errors in the results of both software tools, OptiCF and BirdcageBuilder, increase when the desired resonance frequency is higher. However, the increase in the error of the BirdcageBuilder results is significantly greater than that of OptiCF. It is important to note that the tolerance of the capacitance values must also be taken into consideration when observing the error percentage results. For example, a capacitance value of $1 \mathrm{pF}$ used in the low-pass birdcage coil measurements has a
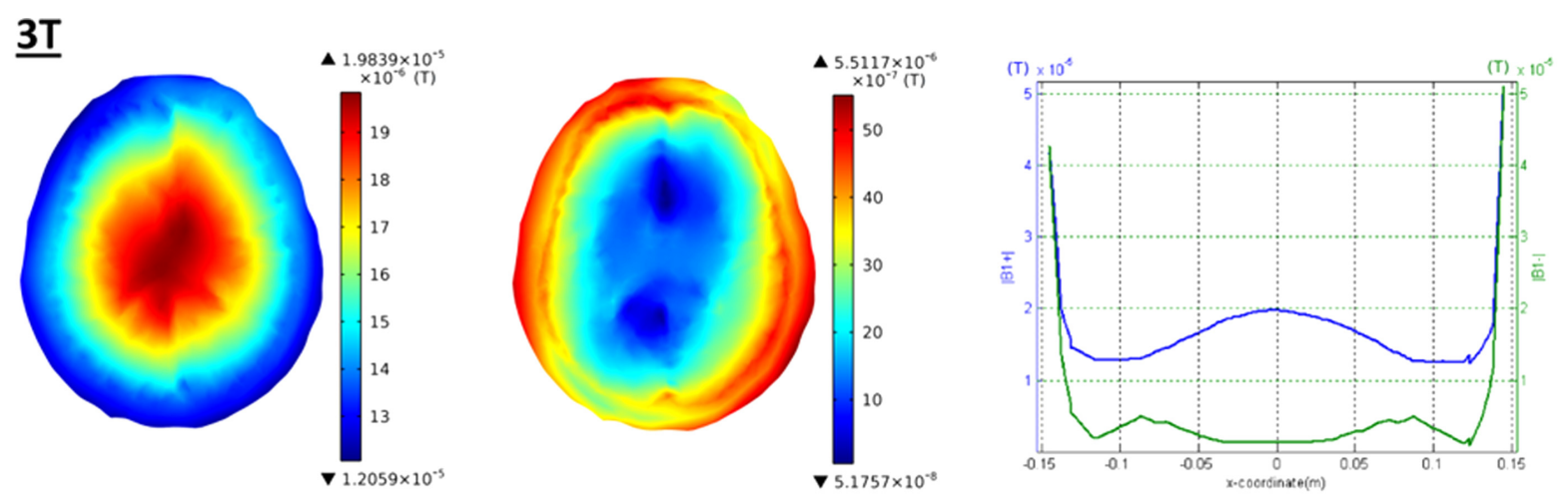

프
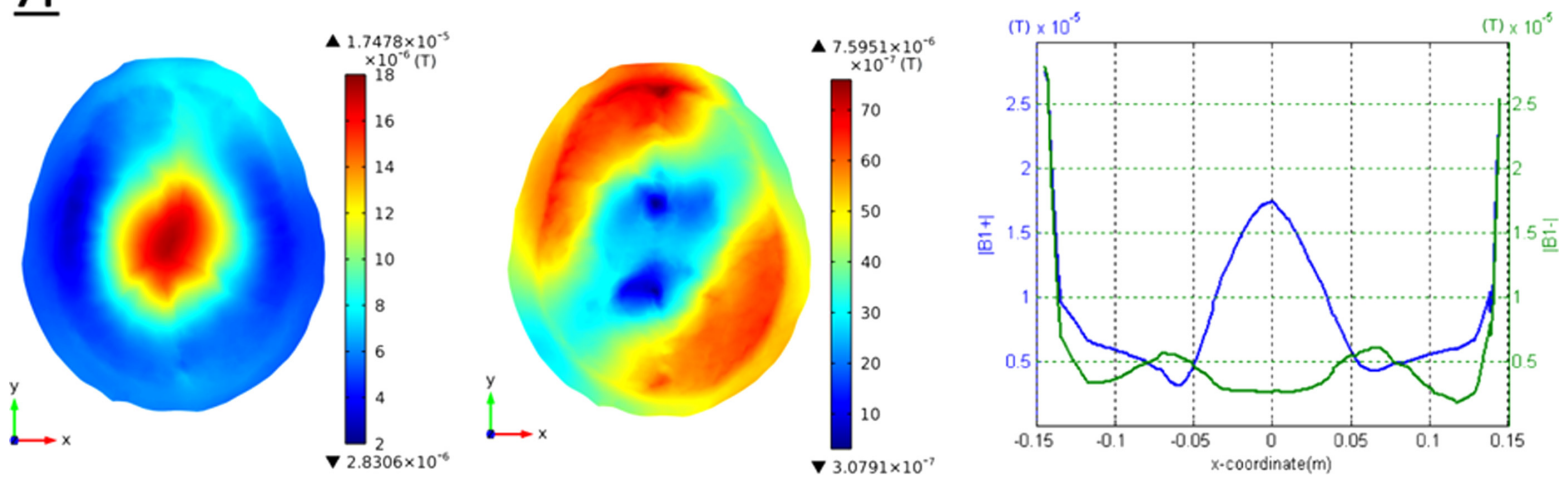

Figure 12 Time-harmonic electromagnetic field solutions of the high-pass birdcage head coil loaded with human head at $3 \mathrm{~T}$ and 7T. (left) $\left|B_{1}^{+}\right|$image, (middle) $\left|B_{1}^{-}\right|$images at the $x$ $y$ plane $(z=0)$, (right) $\left|B_{1}^{+}\right|$and $\left|B_{1}^{-}\right|$along the $(x, y=0, z=0)$ line inside the birdcage coil. 

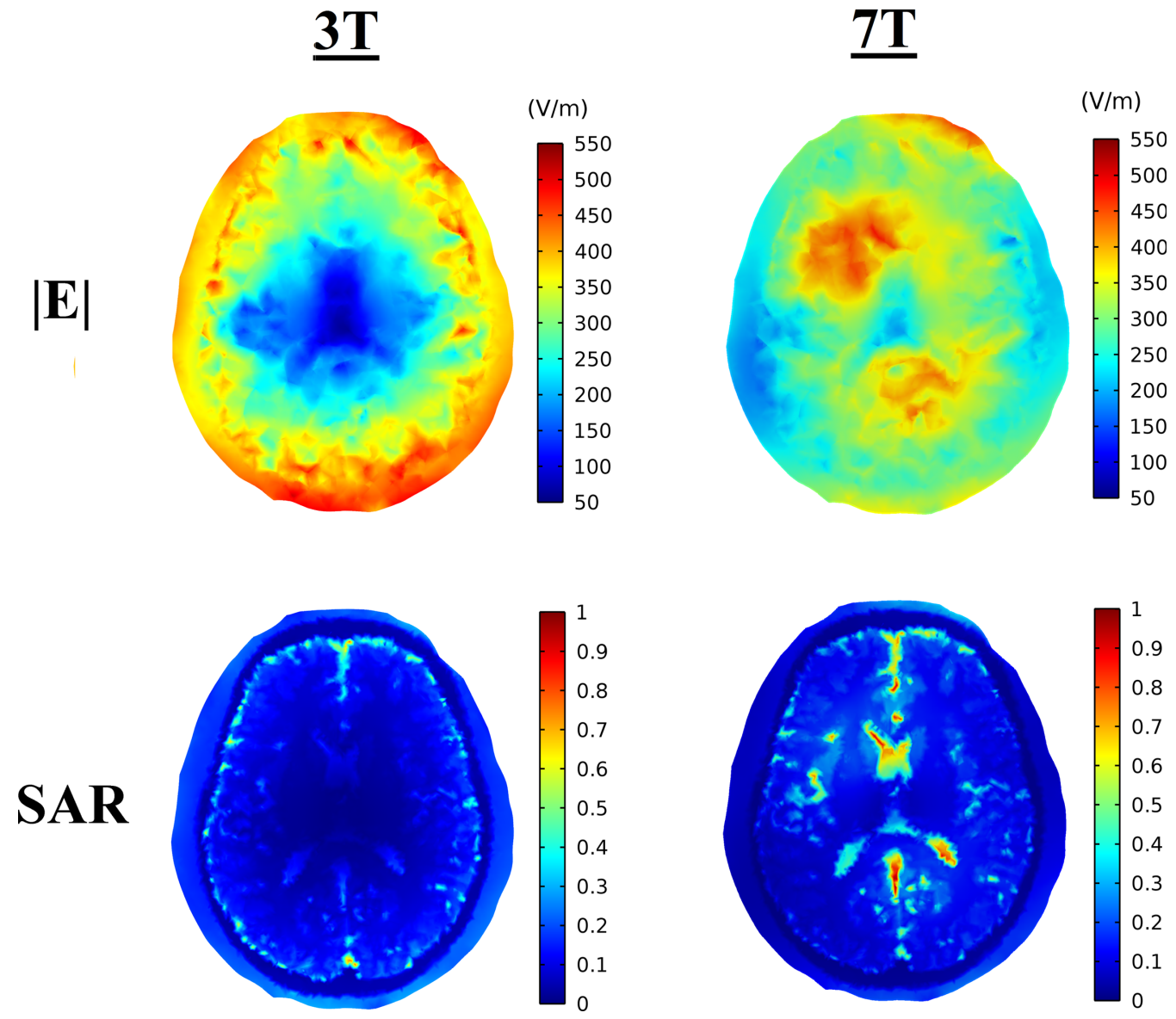

Figure 13 Magnitude of the electric field and normalized SAR images of the human head model at the central slice $(z=0)$ for $3 \mathrm{~T}$ and $7 \mathrm{~T}$.

tolerance of $\pm 0.25 \mathrm{pF}$ which yields $\pm 25 \%$ error to the results inherently. The other important point is that for relatively low frequencies, OptiCF and BirdcageBuilder find close capacitance values, which indicates that using the lumped circuit element model is valid at those frequencies. However, as the frequency increases at which the wavelengths are comparable with the coil dimensions lumped circuit element models fail and electromagnetic field

Table 3 Experimentally Used Capacitance Values and Calculated Capacitance Values Using OptiCF and BirdcageBuilder for Hand-Made Low-Pass Birdcage Coil

\begin{tabular}{lcrr}
\hline $\begin{array}{l}\text { Frequency } \\
(\mathrm{MHz})\end{array}$ & $\begin{array}{c}C_{\exp } \\
(\mathrm{pF})\end{array}$ & $\begin{array}{c}C_{\mathrm{BB}} \\
(\mathrm{pF})\end{array}$ & $\begin{array}{r}C_{\mathrm{opt}} \\
(\mathrm{pF})\end{array}$ \\
\hline 60.75 & 47 & 43.87 & 44.42 \\
122.11 & 10 & 10.86 & 10.46 \\
211.3 & 3.3 & 3.63 & 3.51 \\
255.2 & 1.8 & 2.49 & 1.92 \\
335.7 & 1 & 1.44 & 0.84 \\
\hline
\end{tabular}

calculation based proposed method gives more accurate results.

Second, resonant modes of the birdcage coils calculated using RM-FDA ( $f_{\text {RM-FDA }}$ ) were compared with the resonant modes that are experimentally $\left(f_{\text {exp }}\right)$ measured for five different capacitance values. These measured and calculated resonant frequencies for low-pass and high-pass birdcage coils are given

Table 4 Experimentally Used Capacitance Values and Calculated Capacitance Values Using OptiCF and BirdcageBuilder for Hand-Made high-Pass Birdcage Coil

\begin{tabular}{lccc}
\hline $\begin{array}{l}\text { Frequency } \\
(\mathrm{MHz})\end{array}$ & $\begin{array}{c}\text { Cexp } \\
(\mathrm{pF})\end{array}$ & $\begin{array}{c}\mathrm{CBB} \\
(\mathrm{pF})\end{array}$ & $\begin{array}{c}\text { Copt } \\
(\mathrm{pF})\end{array}$ \\
\hline 75.25 & 100 & 99.27 & 100.34 \\
131.4 & 30 & 32.56 & 32.3 \\
182.5 & 15 & 16.88 & 16.03 \\
245.0 & 7.5 & 9.36 & 8.65 \\
334.26 & 3.3 & 5.03 & 4.2 \\
\hline
\end{tabular}




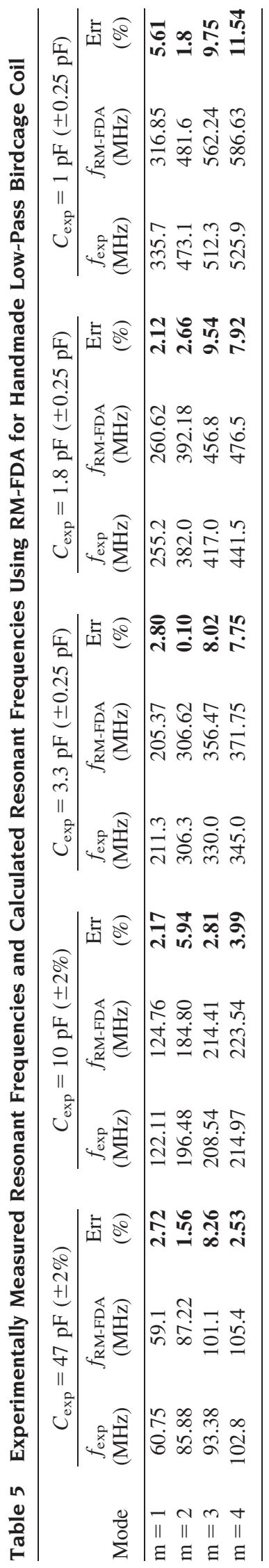

with the corresponding percent errors in Tables 5 and 6 , respectively.

We have made first order regression analysis to observe the relation between the calculated resonant frequencies (dependent variable) and measured resonance frequencies (independent variable). For the low-pass birdcage coil, considering only the data for the $47 \mathrm{pF}$ and $10 \mathrm{pF}$ capacitors (which have low tolerance values of $\pm 2 \%$ ), the slope of the regression line is found to be $1(0.8952,1.105)$ and the offset is found to be $1.892(-13.58,17.37) \mathrm{MHz}$ (The numbers inside the parentheses are 95\% confidence interval boundaries). This means that for these capacitors our calculated frequency values represent the measured values very closely. When all data from all capacitors are included for the low-pass birdcage coil, the slope and the offset become $1.096(1.047,1.146)$ and $-14.42(-30.2$, 1.355) $\mathrm{MHz}$, respectively. These results indicate that having a slope of one has a probability of less than $5 \%$ and that there is a systematic difference between calculated and measured frequencies. We think the reason is that the tolerance of the $C_{\text {exp }}=3.3,1.8$, and $1 \mathrm{pF}$ capacitors are high introducing high variation to the measured frequencies. For the high-pass birdcage coil, considering the data for $C_{\exp }=100,30$, and $15 \mathrm{pF}$ capacitors, the slope and offset of the regression line are found to be $1.065(1.03,1.099)$ and $-3.31(-7.201,0.5806)$ $\mathrm{MHz}$, respectively. When all data for the high-pass case are included, we obtain the slope and offset as $1.086(1.064,1.109)$ and $-6.011(-10.02,-2)$ $\mathrm{MHz}$, respectively. In interpreting the results of the regression analyses one must consider that in addition to errors due to capacitance tolerances, there are also errors in measuring resonant modes using the network analyzer and computation errors in the finite element analysis.

\section{DISCUSSION}

In this study, FEM based methods have been proposed in order to make accurate simulation, design, and resonant mode analysis of loaded and unloaded RF birdcage coils at high frequency using COMSOL Multiphysics. To apply these methods conveniently and using the user-specified parameters, two software tools, RM-FDA and OptiCF, were developed in MATLAB.

Using RM-FDA, time-harmonic electromagnetic field analysis of RF birdcage coils can be made for any scenario: loaded or unloaded case, and linear or quadrature drive. Especially at higher 


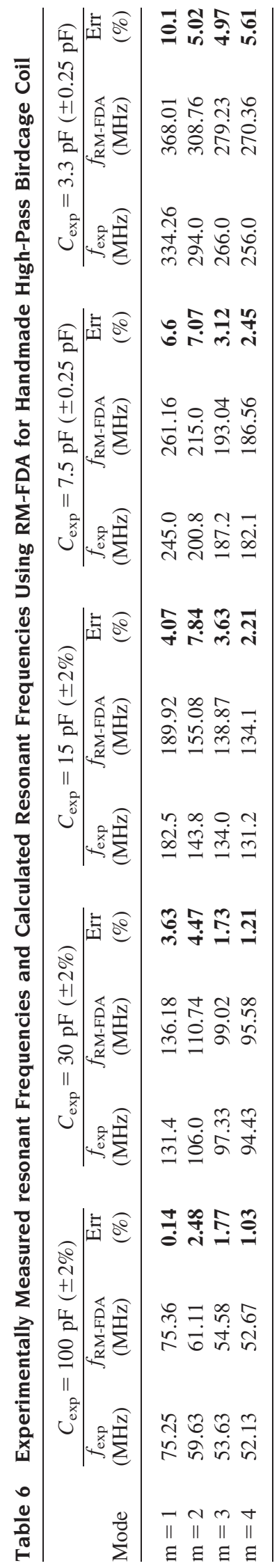

frequencies these simulations are important in terms of understanding the interactions between the RF fields and the object inside the coils. Furthermore, using RM-FDA, resonant modes of the birdcage coil and their associated fields can be directly calculated. Since RM-FDA uses eigenfrequency analysis for finding the resonant frequencies, it is much faster than finding them using a frequency sweep method. Thus, using resonant mode analysis of the RM-FDA tool one can quickly observe the effect of loading on the resonance frequencies and their associated fields. For the unloaded case, $B_{1}^{+}$ field of one of the calculated resonance frequencies is homogenous, and this corresponds to the desired frequency used in MRI. For the loaded case, on the other hand, $B_{1}^{+}$field of the desired mode may not be homogenous due to the presence of the object and also the resonance frequency changes when compared to the resonance frequency in the unloaded case. For loaded 7T resonant mode analysis, it is observed that the solver finds other resonant modes in addition to the unloaded coil resonant modes. These additional resonant modes appear most likely due to the fact that the wavelength of electromagnetic waves in the brain tissue at $300 \mathrm{MHz}(\sim 7 \mathrm{~T})$ is in the order of $10 \mathrm{~cm}$, which is comparable to the dimensions of the head. Investigation of these resonant modes and their potential to generate a hot spot in the brain will be the subject of further studies.

For time-harmonic electromagnetic analysis and resonant mode analysis, RF module of COMSOL Multiphysics was used. Time-harmonic electromagnetic analysis, that is, solution of a forward problem, for one frequency takes about 3 minutes for the birdcage coil shown in Fig. 8 for which the FEM mesh has 1885626 degrees of freedom for the unloaded case. For the loaded case, the solution takes about 13 minutes for 2026578 degrees of freedom. In loaded case simulations, most of the time is spent on interpolating the material properties (conductivity and permittivity) to mesh nodes. For the same geometry, resonant mode analysis takes about 18 minutes for the unloaded case and 30 minutes for the loaded case. As previously discussed, direct determination of resonant modes based on eigenfrequency analysis is much faster than their determination through a frequency sweep method.

The other software tool, OptiCF, is used to calculate the capacitance value necessary to make the coil resonate at the desired frequency. This calculation is based on optimization and is made using two different objective functions: $|Z|$ and the variance 
of $\left|B_{1}^{+}\right|$. Optimization module of COMSOL Multiphysics uses gradient based optimization method (44) and therefore objective functions must be continuous and differentiable with respect to the control variable. In this study, both the $|Z|$ and variance of $\mid$ $B_{1}^{+} \mid$satisfy these requirements with respect to the capacitance value. However, the solution may converge to local minima if the capacitance range is not defined correctly. For this purpose, we propose the use of variance of $\left|B_{1}^{+}\right|$to define the capacitance range correctly and make the optimization using $|Z|$ as an objective function. Especially at high frequencies, using OptiCF will decrease the duration of tuning and matching procedures by calculating an accurate initial capacitance value.

Maximizing the magnitude of the port impedance in the method of tuning the birdcage coil seems confusing, since minimizing the $S_{11}$ of the coil is more commonly used by coil designers for this purpose. In our opinion, for finding the most accurate initial capacitance value for the homogenous mode, use of the port impedance seems more appropriate, since the matching of the coil is not performed in the simulations. When matching is not considered, variation of $\mathrm{S}_{11}$ with frequency does not depict sharp minimums at the resonant modes, and therefore use of $S_{11}$ for numerical optimization purposes will not be suitable. However, in practice, since matching must also be performed, $\mathrm{S}_{11}$ measurements need to be taken into consideration. Therefore, resonant frequencies that we have found in the simulations were compared to the experimentally observed resonant frequencies obtained from $S_{11}$ measurements.

In this study, capacitances on the rungs or end rings were assumed to have the same value, and therefore optimization was made for the unloaded case using that capacitance value. In the loaded case, the homogeneity of the magnetic field deteriorates. However, using different capacitances on the individual rungs or end rings, and by making the optimization for all the capacitance values, one may obtain a more homogenous magnetic field for the loaded case.

Rungs and end rings were modeled by rectangular strips in this study. Instead of rectangular strips, wires (cylindrical rods) may also be used, especially in low-field MR coils, because they have a higher quality factor than the rectangular strips (45). At high frequencies, on the other hand, sample losses are dominant, and the use of wires may not significantly contribute to the quality factor of the coil. The users, who may wish to use wire conductors in the proposed software tools, may still use "equivalent" rectangular strips which have the same inductance values as the wire conductors. The rela- tion between the width of a rectangular strip and the radius of a wire - in order to achieve the same inductance value - is given in (45). It is also not difficult to modify the source code to incorporate wire model options for rungs and end rings.

For the coils used in high-field MRI, it is common practice to ensure that uninterrupted conductive segments are much smaller than the wavelength in air. This can be achieved by designing band-pass birdcage coils and by placing more than one capacitors to the rung elements to decrease the length of uninterrupted conductive segments. For the proposed software tools, in their current form, these designs cannot be performed directly but the users can modify the source codes and can adapt the software tool according to their purpose of use. Similarly, designing of high-field MRI coils, such as TEM resonator, or multiple independently driven transmit elements in the light of proposed methods will be the subject of future studies.

\section{ACKNOWLEDGMENT}

This work was supported by The Scientific and Technological Research Council of Turkey (TUBITAK) under grant 111E090.

\section{REFERENCES}

1. Hayes CE, Edelstein WA, Schenck JF, Mueller OM, Eash M. 1985. An efficient, highly homogeneous radiofrequency coil for Whole-body NMR imaging at 1.5 T. J Magn Reson 63:622-628.

2. Tropp, J. 1989. The theory of the bird-cage resonator. J Magn Reson (1969) 82 no. 1:51-62.

3. Leifer MC. 1997. Resonant modes of the birdcage coil. J Magn Reson 124:51-60.

4. Pascone R, Garcia TFBJ, Vullo RZT, Cahill P. 1991. Generalized electrical analysis of low-pass and high-pass birdcage resonators. Magn Reson Imaging 9:395-408.

5. Giovannetti G, Landini L, Santarelli MF, Positano V. 2002. A fast and accurate simulator for the design of birdcage coils in MRI. Magn Reson Mater Phys Biol Med 15:36-44.

6. Chin CL, Collins CM, Li S, Dardzinski BJ, Smith MB. 2002. BirdcageBuilder: design of Specified-geometry birdcage coils with desired current pattern and resonant frequency. Concept Magn Reson 15:156-163.

7. Ibrahim TS, Lee R, Baertlein BA, Kangarlu A, Robitaille PML. 2000. Application of finite difference time domain method for the design of birdcage RF head coils using multi-port excitations. Magn Reson Imaging 18:733-742. 
8. Ibrahim TS, Lee R, Baertlein BA, Yu Y, Robitaille PML. 2000. Computational analysis of the high pass birdcage resonator: finite difference time domain simulations for high-field MRI. Magn Reson Imag 18: 835-843.

9. Ibrahim TS, Kangarlu A, Chakeress DW. 2005. Design and performance issues of RF coils utilized in ultra high field MRI: experimental and numerical evaluations. Biomed Eng IEEE Trans 52:1278-1284.

10. Collins CM, Li S, Smith MB. 1998. SAR, B1 field distributions in a heterogeneous human head model within a birdcage coi. Magn Reson Med 40:847-856.

11. Collins CM, Smith MB. 2001. Calculations of b1 distribution, SNR, and SAR for a surface coil adjacent to an anatomically-accurate human body model. Magn Reson Med 45:692-699.

12. Ibrahim TS, Lee R, Baertlein BA, Robitaille PML. 2001. B1 field homogeneity and SAR calculations for the birdcage coil. Phys Med Biol 46:609

13. Ibrahim TS, Mitchell C, Abraham R, Schmalbrock P. 2007. In-depth study of the electromagnetics of ultrahigh-field MRI. NMR Biomed 20:58-68.

14. Guclu C, Kashmar G, Hacinliyan A, Nalcioglu O. 1997. An FEM approach for the characterization of the RF field homogeneity at high field.. Magn Reson Med 37:76-83.

15. Jin J, Chen J. 1997. On the SAR and field inhomogeneity of birdcage coils loaded with the human head. Magn Reson Med 38:953-963.

16. Chen JH, Jeng SK, Lin FH, Kuan WP. 1999. Quantitative analysis of magnetic resonance radio-frequency coils based on method of moments. IEEE Trans Magnetics 35, no. 4.

17. Kumar A, Bottomley PA. 2006. Optimizing the intrinsic signal-to-noise ratio of MRI strip detectors. Magn Reson Med 56:157-166.

18. Lin, FH, Kuan, WP, Jeng SK, Chen, JH. 1999. Quantitative spectral/spatial analysis of phased array coil in magnetic resonance imaging based on method of moment. IEEE Trans Med Imaging 18, no. 12:1129_ 1137.

19. Chen J, Feng Z, Jin JM. 1998. Numerical simulation of SAR and B1-field inhomogeneity of shielded RF coils loaded with the human head. IEEE Trans Biomed Eng 45:650-659.

20. Li BK, Liu F, Crozier S. 2005. Focused, eightelement transceive phased array coil for parallel magnetic resonance imaging of the chest-theoretical considerations. Magn Reson Med 53, no. 6:12511257.

21. Li BK, Liu F, Weber E, Crozier S. 2009. Hybrid numerical techniques for the modelling of radiofrequency coils in MRI. NMR Biomed 22:937-951.

22. Xin X, Wang D, Han J, Feng Y, Feng Q, Chen W. 2012. Numerical optimization of a three-channel radiofrequency coil for open, vertical-field, MRguided, focused ultrasound surgery using the hybrid method of moment/finite difference time domain method. NMR Biomed 25:909-916.

23. Collins CM. 2009. Numerical field calculations considering the human subject for engineering and safety assurance in MRI. NMR Biomed 22:919-926.

24. Collins CM, Wang Z. 2011. Calculation of radiofrequency electromagnetic fields and their effects in MRI of human subjects. Magn Reson Med 65:14701482.

25. Ibrahim TS, Hue YK, Tang L. 2009. Understanding and manipulating the RF fields at high field MRI. NMR Biomed 22:927-936.

26. Katscher U, Börnert P, Leussler C, Brink JSvd. 2003. Transmit SENSE. Magn Reson Med 49:144-150.

27. Berg CATVd, Bergen Bvd, Kamer JBVd, Raaymakers BW, Kroeze H, Bartels LW, et al. 2007. Simultaneous b1 + homogenization and specific absorption rate hotspot suppression using a magnetic resonance phased array transmit coil. Magn Reson Med 57:577-586.

28. Vaughan T, DelaBarre L, Snyder C, Tian J, Akgun C, Shrivastava D, et al. 2006. 9.4T human MRI: preliminary results. Magn Reson Med 56:12741282.

29. Metzger GJ, Snyder C, Akgun C, Vaughan T, Ugurbil K, Moortele PFVd. 2008. Local b1+ shimming for prostate imaging with transceiver arrays at $7 \mathrm{~T}$ based on subject-dependent transmit phase measurements. Magn Reson Med 59:396-409.

30. Wolf S, Diehl D, Gebhardt M, Mallow J, Speck O. 2013. SAR simulations for high-field MRI: how much detail, effort, and accuracy is needed? Magn Reson Med 69:1157-1168.

31. Jin J, Liu F, Weber ve E, Crozier S. 2012. Improving SAR estimations in MRI using subject-specific models. Phys Med Biol cilt 57, no. 24:8153-8171.

32. Duan Y, Peterson BS, Liu F, Brown TR, Ibrahim TS, Kangarlu A. 2009. Computational and experimental optimization of a double-tuned 1H/31P four-ring birdcage head coil for MRS at 3T. J Magn Reson Imaging 29:13-22.

33. Gurler N, Ider YZ. FEM based design and simulation tool for MRI birdcage coils including eigenfrequency analysis, in COMSOL Proc., Milan, Italy, 2012.

34. Duan Y, Ibrahim TS, Peterson BS, Liu F, Kangarlu A. 2008. Assessment of a PML boundary condition for simulating an MRI radio frequency coil. Int $\mathrm{J}$ Antennas Propagation 2008, 2008:2008

35. COMSOL AB, RF Module User's Guide, Version October 2011 COMSOL 4.2a, 2011.

36. Katscher U, Voigt T, Findeklee C, Vernickel P, Nehrke K, Dossel O. 2009. Determination of electric conductivity and local SAR via b1 mapping. Med Imaging, IEEE Trans 28:1365-1374.

37. Zhang X, Zhu S, He B. 2010. Imaging electric properties of biological tissues by RF field mapping in MRI. Med Imaging, IEEE Trans 29:474-481. 
38. Hafalir FS, Oran OF, Gurler N, Ider YZ. 2014. Convection-reaction equation based magnetic resonance electrical properties tomography (cr-MREPT). Med Imaging, IEEE Trans 33:777-793.

39. Gurler N, Hafalir FS, Oran OF, Ider YZ. A New Accurate FEM based Optimization Method for Birdcage Coil Design at High Field Strength, in Proc. 21th Annual Meeting ISMRM, Salt Lake City, Utah, USA, 2013.

40. BrainWeb: Simulated Brain Database, Available at: http://brainweb.bic.mni.mcgill.ca/brainweb/.

41. Aubert-Broche B, Evans AC, Collins L. 2006. A new improved version of the realistic digital brain phantom. NeuroImage 32:138-145.
42. Fang Q. Iso2mesh: a 3D surface and volumetric mesh generator for MATLAB/Octave. Available at: http:// iso2mesh.sourceforge.net/.

43. Fang Q, Boas D. Tetrahedral mesh generation from volumetric binary and gray-scale images, in Proceedings of IEEE International Symposium on Biomedical Imaging, Boston, 2009.

44. Gill P, Murray W, Saunders M. 2005. SNOPT: an SQP algorithm for Large-scale constrained optimization. SIAM Rev 47:99-131.

45. Giovannetti G, Hartwig V, Landini L, Santarelli MF. 2010. Low-field MR coils: comparison between strip and wire conductors. Appl Magn Reson 39:391-399. 